\title{
REGULARIZATION BY NOISE AND FLOWS OF SOLUTIONS FOR A STOCHASTIC HEAT EQUATION
}

\author{
By OLEG ButKovsKY ${ }^{1}$ AND LeONID MYTNIK ${ }^{2}$ \\ Technion-Israel Institute of Technology
}

Motivated by the regularization by noise phenomenon for SDEs, we prove existence and uniqueness of the flow of solutions for the non-Lipschitz stochastic heat equation

$$
\frac{\partial u}{\partial t}=\frac{1}{2} \frac{\partial^{2} u}{\partial z^{2}}+b(u(t, z))+\dot{W}(t, z),
$$

where $\dot{W}$ is a space-time white noise on $\mathbb{R}_{+} \times \mathbb{R}$ and $b$ is a bounded measurable function on $\mathbb{R}$. As a byproduct of our proof, we also establish the so-called path-by-path uniqueness for any initial condition in a certain class on the same set of probability one. To obtain these results, we develop a new approach that extends Davie's method (2007) to the context of stochastic partial differential equations.

1. Introduction. This work deals with the uniqueness theory for stochastic heat equations of the following form:

$$
\begin{aligned}
\frac{\partial u}{\partial t} & =\frac{1}{2} \frac{\partial^{2} u}{\partial z^{2}}+b(u(t, z))+\dot{W}(t, z), \quad t \geq 0, z \in \mathbb{R}, \\
u(0, z) & =q(z),
\end{aligned}
$$

where $\dot{W}$ is a Gaussian space-time white noise on $\mathbb{R}_{+} \times \mathbb{R}, b$ is a bounded Borel measurable function on $\mathbb{R}$ and $q$ is a Borel measurable function on $\mathbb{R}$ satisfying certain growth conditions. To be more precise, we are going to construct the flow of solutions to (1.1), which is indexed by initial conditions $q$; we will establish uniqueness of the flow and show that in fact the flow can be constructed in a PDE sense on a set of full probability measure.

Equation (1.1) has been extensively studied in the SPDE literature. The strong existence and uniqueness (in a probability sense) to that equation has been shown

Received November 2016; revised February 2018.

${ }^{1}$ Supported in part by Israel Science Foundation Grants 1325/14 and 1131/14, Russian Foundation for Basic Research Grant 13-01-00612-a, DFG Research Unit FOR 2402, NSF Grant DMS-1440140 while OB was in residence at the Mathematical Sciences Research Institute (MSRI) in Berkeley, California, during the Fall 2015 semester, and a Technion fellowship.

${ }^{2}$ Supported in part by Israel Science Foundation Grant 1131/14.

MSC2010 subject classifications. 60H15, 35R60, 60H25.

Key words and phrases. Regularization by noise, stochastic heat equation, path-by-path uniqueness, stochastic flow of solutions. 
by Gyöngy and Pardoux in [12] for bounded $b$ and in [13] for some locally unbounded $b$. Later, in [1], the results were extended to the equations with the multiplicative noise. Note that in the above references the equations are defined for the spatial variable $z \in[0,1]$, but the results could be easily extended to our setting of $z \in \mathbb{R}$.

The strong uniqueness for (1.1) represents a phenomenon that is called "regularization by noise." This is the property that roughly speaking can be formulated as follows: deterministic equation without noise might not have uniqueness or existence property; however, whenever the equation is perturbed by noise it has a unique solution; see the related discussion in a recent book of Flandoli [8]. This is the situation with (1.1): clearly, one cannot make a general claim that equation (1.1) without noise at the right-hand side has a unique solution whenever $b$ is not Lipschitz, whereas, as we mentioned above, with the noise, uniqueness holds for a large class of drifts $b$. Note that whenever we say that there exists a unique strong solution to (1.1) we mean by this that on some filtered probability space $\left(\Omega, \mathcal{F},\left(\mathcal{F}_{t}\right)_{t \geq 0}, \mathrm{P}\right)$ there exists a unique adapted strong solution to that equation. That, in fact, implies that regularization by noise phenomenon happens in probability sense, as a regularization for Itô-Walsh stochastic equation.

On the other hand, one can ask the question whether the regularization effect takes place in a purely PDE setting. That is, one is interested whether it is possible to find a set $\Omega^{\prime} \subset \Omega$ of full probability such that for almost every $\omega \in \Omega^{\prime}$, given the path

$$
(t, z) \mapsto V(t, z, \omega):=\int_{0}^{t} \int_{\mathbb{R}} p_{t-t^{\prime}}\left(z-z^{\prime}\right) W\left(d t^{\prime}, d z^{\prime}, \omega\right)
$$

(see the discussion in the beginning of Section 2 for the precise definition of $V$ ) equation (1.1) in the integral, or so-called, mild form (see equation (2.1) below), has a unique solution. Due to Flandoli's definition, we will call the uniqueness of such kind the path-by-path uniqueness; see [8], Definition 1.5 and the discussion in [8], Section 1.3.3.

The problem of path-by-path uniqueness is interesting in itself; however, it is closely related to another interesting question: existence and uniqueness of the flow of solutions indexed by initial conditions $q$ of the equation. To the best of our knowledge, not much is known about existence and uniqueness of flows for SPDEs. Even if the drift and diffusion are very smooth functions, only the local flow property was established in [14], Corollary 1.10. If the drift is Lipschitz and the diffusion coefficient is linear, the flow property was proved in [11]; see also [4] for related results. Linear systems were considered earlier by Flandoli [7]. We are not aware of any results in the literature concerning the case of non-Lipschitz coefficients; in the current paper, we study an SPDE with a non-Lipschitz drift and an additive noise.

The question of regularization by noise for SDEs has been studied much more extensively. In particular, the following SDE has been thoroughly investigated:

$$
d X_{t}=b\left(X_{t}\right) d t+d B_{t},
$$


where $b$ is a measurable function and $B$ is a $d$-dimensional Brownian motion defined on a filtered probability space $\left(\Omega, \mathcal{F},\left(\mathcal{F}_{t}\right)_{t \geq 0}, \mathrm{P}\right)$. First, it was derived by Zvonkin in [27] for $d=1$, that the above equation has a unique strong solution for a bounded measurable $b$. Then this result was generalized by Veretennikov in [23] for the multidimensional case, and later it was extended by Krylov and Röckner in [17] for the case of locally unbounded $b$ under some integrability condition. The flow property of solutions to (1.2) was also established under essentially the same integrability condition; see $[6,9]$ and [26] for the case of nonconstant diffusion coefficients. Note that the definition of stochastic flow in the above references requires that the solution $\left\{X_{t}, t \geq 0\right\}$ is adapted with respect to the filtration $\mathcal{F}_{t}$. In particular, the strong uniqueness, is by definition, the uniqueness among the adapted solutions. All the proofs use a Zvonkin-type transformation [27] that allows either to eliminate the "nonregular" drift or to make it more regular. For the related recent interesting works on flows of SDEs, see also [19, 21].

If one asks the path-by-path uniqueness for (1.2), then the first result in this direction has been achieved by Davie in [5], who showed it for a fixed initial condition $x$. Later the result has been generalized by Shaposhnikov in [22], who established path-by-path uniqueness of solutions simultaneously for all initial conditions. Shaposhnikov also developed a new method that is based on the flow construction of Fedrizzi and Flandoli [6]. Recently, the regularization by noise has been constructed also for equations driven by other types of noises, for example, Lévy noises: see Priola [20], where Shaposhnikov's method is used. We would also like to mention a paper by Catellier and Gubinelli [3] where a number of very interesting results concerning regularization by noise and path-by-path uniqueness for ODEs were achieved. Recently, some results related to path-by-path uniqueness for Hilbert space-valued SDEs were obtained in [25] for fixed initial conditions; however, the flow property is not obtained in that paper.

Now if we get back to our SPDE setting, we can say outright that we do not have the luxury of having a convenient Zvonkin-type transformation. That is why we, in a sense, use the reverse argument: we first show path-by-path uniqueness together with some continuity with respect to initial conditions and based on this we show existence and uniqueness of the flow. To push the argument through, we develop a new method that extends Davie's approach to the infinite-dimensional case. We believe that our method of proving existence of the flow is of independent interest.

In the next section, we will present the main results of the paper.

2. Main results. We study a one-dimensional stochastic heat equation on $\mathbb{R}$ with a drift (1.1). Let $\left(\Omega, \mathcal{F},\left(\mathcal{F}_{t}\right)_{t \geq 0}, \mathrm{P}\right)$ be a probability space. Let $\dot{W}$ be a spacetime white noise on this space adapted to the filtration. Let $p$ be a standard heat kernel

$$
p_{t}(z)=\frac{1}{\sqrt{2 \pi t}} \exp \left(-z^{2} / 2 t\right), \quad t>0, z \in \mathbb{R}
$$


and $V$ be a convolution of the heat kernel $p$ with the white noise $\dot{W}(\cdot, \cdot, \omega)$, that is,

$$
V(s, t, z, \omega):=\int_{s}^{t} \int_{\mathbb{R}} p_{t-t^{\prime}}\left(z-z^{\prime}\right) W\left(d t^{\prime}, d z^{\prime}\right), \quad t \geq 0, s \in[0, t], z \in \mathbb{R} .
$$

In case $s=0$ for brevity, we drop the first index and write $V(t, z, \omega):=$ $V(0, t, z, \omega)$. Further, we will frequently omit $\omega$ from the notation. Later on, in Lemma 4.7 we will show existence of a modification of $V$ that is almost surely jointly continuous in $(s, t, z)$; with some abuse of notation this modification will be denoted by the same symbol $V$. As usual, here and in the sequel we use the convention that $\int p_{0}(x-y) f(y) d y:=f(x)$ for any measurable function $f$.

We say that a random function $u$ solves (1.1) in the path-by-path sense if $u(0, z)=q(z)$ and for P-almost surely $\omega$ the following holds for any $t>0, z \in \mathbb{R}$ :

$$
\begin{aligned}
u(t, z, \omega)= & \int_{\mathbb{R}} p_{t}\left(z-z^{\prime}\right) q\left(z^{\prime}\right) d z^{\prime} \\
& +\int_{0}^{t} \int_{\mathbb{R}} p_{t-t^{\prime}}\left(z-z^{\prime}\right) b\left(u\left(t^{\prime}, z^{\prime}, \omega\right)\right) d z^{\prime} d t^{\prime} \\
& +V(0, t, z, \omega) .
\end{aligned}
$$

We will also consider a stochastic heat equation that starts with the initial condition $q$ at time $s \geq 0$ :

$$
\begin{aligned}
u(t, z, \omega)= & \int_{\mathbb{R}} p_{t-s}\left(z-z^{\prime}\right) q\left(z^{\prime}, \omega\right) d z^{\prime} \\
& +\int_{s}^{t} \int_{\mathbb{R}} p_{t-t^{\prime}}\left(z-z^{\prime}\right) b\left(u\left(t^{\prime}, z^{\prime}, \omega\right)\right) d z^{\prime} d t^{\prime} \\
& +V(s, t, z, \omega), \quad t>s, z \in \mathbb{R}, \\
u(s, z, \omega)= & q(z, \omega), \quad z \in \mathbb{R} .
\end{aligned}
$$

Sometimes when there is an ambiguity we denote a solution to (2.2) by $u_{s, q}(t, z$, $\omega)$, thus emphasizing the initial conditions. We see that for $s=0$ (2.2) is just (2.1). We have to analyze $u_{s, q}$ for $s \geq 0$ (rather than just at $s=0$ ) in order to prove the existence of the flow; see the proof of Theorem 2.2(a) below.

Note that the difference between the definition given above and the standard one (see, e.g., [16], Definition 6.3) is that we do not require adaptiveness of the solution $u$ to the filtration generated by $\dot{W}$. Instead of it, for each fixed $\omega \in \Omega$ we treat equation (2.2) separately as a deterministic PDE with a forcing term $V(s, \cdot, \cdot, \omega)$.

Let us now present the main results of the paper. First we define a class of functions that we take as initial conditions to (1.1).

\section{DEFINITION 2.1.}

1) Let $\mu \geq 0$. We say that a measurable function $f: \mathbb{R} \rightarrow \mathbb{R}$ belongs to the class $\mathbf{B}(\mu)$, if there exists a constant $C>0$ such that $|f(z)| \leq C\left(|z|^{\mu} \vee 1\right)$ for $z \in \mathbb{R}$. 
2) We say that a function $f: \mathbb{R} \rightarrow \mathbb{R}$ belongs to the class $\mathbf{B}(0+)$, if $f$ belongs to the class $\mathbf{B}(\varepsilon)$ for all $\varepsilon>0$.

For brevity, the class $\mathbf{B}(0)$ of measurable bounded functions on $\mathbb{R}$ will be denoted just by $\mathbf{B}$. If $f \in \mathbf{B}$, we define $\|f\|_{\infty}:=\sup _{z \in \mathbb{R}}|f(z)|$.

Our first result proves existence and path-by-path uniqueness (see brief discussion on this concept in the Introduction) of solutions to (1.1) on some "good" set of full probability measure simultaneously for all initial conditions in $\mathbf{B}(0+)$ and all starting times $s \geq 0$.

THEOREM 2.1. Let $b \in \mathbf{B}$. There exists a set $\Omega^{\prime}=\Omega^{\prime}(b) \subset \Omega$ with the following properties:

1) $\mathrm{P}\left(\Omega^{\prime}\right)=1$.

2) Let $\omega \in \Omega^{\prime}$. Then for any initial condition $q \in \mathbf{B}(0+)$ and any $s \geq 0$ equation (2.2) has a unique solution. This solution $u_{s, q}(t, \cdot) \in \mathbf{B}(0+)$ for any $t \geq s$.

3) Let $\omega \in \Omega^{\prime}$ and $q_{1}, q_{2} \in \mathbf{B}(0+)$ be two initial conditions. If we have $q_{1}(z)=$ $q_{2}(z)$ Lebesgue-almost everywhere in $z$, then $u_{s, q_{1}}(t, z, \omega)=u_{s, q_{2}}(t, z, \omega)$ for any $s \geq 0, t>s, z \in \mathbb{R}$.

Note that as a class of initial conditions we chose $\mathbf{B}(0+)$ (rather than, e.g., $\mathbf{B}$ ), since $u_{s, q}(t, \cdot) \in \mathbf{B}(0+)$. Thus, if one starts equation (2.2) from an initial condition in $\mathbf{B}(0+)$, then at any $t \geq 0$ the solution to this equation remains in the same class.

SKETCH OF THE PROOF OF THEOREM 2.1. The proof consists of three independent parts. First, in Section 4 we establish a number of useful regularity properties of $V$ (on a certain "good" set) and prove that a certain auxiliary operator is continuous.

Then in Section 5.1 we prove existence of a solution to (2.2). Let $\mathbf{C}_{0}(\mathbb{R})$ be the space of all continuous functions $\mathbb{R} \rightarrow \mathbb{R}$ vanishing at infinity equipped with the standard sup-norm. Recall that it follows from Gyöngy and Pardoux [12] that for any $s \geq 0, q \in \mathbf{C}_{0}(\mathbb{R})$ there exists a set $\Omega_{s, q}$ of probability measure 1 such that on $\Omega_{s, q}$ equation (2.2) has a solution that starts with the initial condition $q$ at time $s$. More precisely, although in [12] the equation is considered on $(t, z) \in \mathbb{R}_{+} \times[0,1]$, the methods that are used in that paper work exactly in the same way for $(t, z) \in$ $\mathbb{R}_{+} \times \mathbb{R}$. Our goal is to show that this "good" set $\Omega_{s, q}$ can be chosen to be the same for all $s \geq 0, q \in \mathbf{B}(0+)$.

To carry out this plan, we fix a countable dense subset $\Xi$ of $\mathbf{C}_{0}(\mathbb{R})$ and a countable dense subset $\Theta$ of $\mathbb{R}_{+}$. Since both $\Xi$ and $\Theta$ are countable, we see that [12] implies that there exists a set $\Omega_{E}$ of probability measure 1 such that for any $\omega \in \Omega_{E}$, $s \in \Theta, q \in \Xi$ equation (2.2) has a solution that starts with the initial condition $q$ at time $s$. Using continuity of a certain integral operator (Lemma 4.10), we will show that there exists a set of full measure $\Omega^{\prime} \subset \Omega_{E}$ such that for any $\omega \in \Omega^{\prime}, s \geq 0$, 
$q \in \mathbf{B}(0+)$ equation (2.2) has a solution that starts with the initial condition $q$ at time $s$.

Finally, in Section 5.2 we prove uniqueness of a solution to (2.2). The proof extensively used smoothing properties of an integral operator involving white noise (Theorem 2.3 and Lemma 5.6). We develop a new approach motivated by the ideas of Davie [5].

The next theorem shows that there exists a unique flow of solutions to equation (1.1) and that this flow is continuous. We will see that this is a direct corollary of existence and path-by-path uniqueness of solutions to (1.1).

THEOREM 2.2. Let $b \in$ B. Let $\Omega^{\prime} \subset \Omega$ be from Theorem 2.1.

(a) [Existence of the flow.] There exists a mapping

$$
(s, t, q, \omega) \mapsto \varphi(s, t, q, \omega)
$$

with values in $\mathbf{B}(0+)$ defined for $0 \leq s \leq t, q \in \mathbf{B}(0+), \omega \in \Omega^{\prime}$ such that:

1. For any $s \geq 0, q \in \mathbf{B}(0+), \omega \in \Omega^{\prime}$ the function $u_{s, q}(t, \cdot):=\varphi(s, t, q, \omega)$ is a unique solution to (2.2) that starts from the initial condition $q$ at time $s$;

2. We have on $\Omega^{\prime}$ for $0 \leq r<s<t$

$$
\varphi(r, t, q, \omega)=\varphi(s, t, \varphi(r, s, q, \omega), \omega)
$$

(b) [Continuity of the flow.] Let $\varphi$ be the mapping defined in Part (a) of the theorem. Let $\left(q_{n}\right)_{n \in \mathbb{Z}_{+}}$be a sequence of functions from $\mathbf{B}(0+)$, that converges Lebesgue-almost everywhere to $q \in \mathbf{B}(0+)$. Assume that there exist constants $C>0, \mu>0$ such that for any $n \in \mathbb{Z}_{+}$one has

$$
\left|q_{n}(z)\right| \leq C\left(|z|^{\mu} \vee 1\right), \quad z \in \mathbb{R} .
$$

Then on $\Omega^{\prime}$ we have for $0 \leq s<t, z \in \mathbb{R}$

$$
\lim _{n \rightarrow \infty} \varphi\left(s, t, q_{n}, \omega\right)(z)=\varphi(s, t, q, \omega)(z) .
$$

Proof of Theorem 2.2(a). By Theorem 2.1, for any $\omega \in \Omega^{\prime}, q \in \mathbf{B}(0+)$, $s \geq 0$ equation (2.2) has a unique solution $u_{s, q}$ that starts with initial condition $q$ at time $s$.

Now for $0 \leq s \leq t, q \in \mathbf{B}(0+), \omega \in \Omega^{\prime}$ define

$$
\varphi(s, t, q, \omega):=u_{s, q}(t, \cdot, \omega) .
$$

Let us check that $\varphi$ satisfies all the properties of the flow formulated in Theorem 2.2(a). The first property is obvious. To check the second property, we fix any $\omega \in \Omega^{\prime}, 0 \leq r<s, q \in \mathbf{B}(0+)$. For $t \geq s$, put $u_{1}(t, \cdot):=\varphi(r, t, q, \omega)$ and 
$u_{2}(t, \cdot):=\varphi(s, t, \varphi(r, s, q, \omega), \omega)$. Note that both $u_{1}$ and $u_{2}$ are solutions to equation (2.2) that starts with initial condition $\varphi(r, s, q, \omega)$ at time $s$. The initial condition $\varphi(r, s, q, \omega)=u_{r, q}(s, \cdot, \omega)$ is in $\mathbf{B}(0+)$ by Theorem 2.1. Therefore, by Theorem 2.1 the solutions $u_{1}$ and $u_{2}$ coincide. Thus,

$$
\varphi(r, t, q, \omega)=\varphi(s, t, \varphi(r, s, q, \omega), \omega),
$$

and $\varphi$ is a flow of solutions to (2.2).

The proof of Theorem 2.2(b) is given in Section 5.3.

The next theorem describes smoothing properties of the noise $V$ that are crucial for the proof of Theorems 2.1 and 2.2. We are interested in the regularity properties of the mapping

$$
(x, t, z) \mapsto \int_{0}^{t} b(V(r, z)+f(r, z)+x) d r,
$$

where $f$ belongs to a certain class of weighted Hölder functions with singularities defined below.

\section{DEFINITION 2.2.}

1) Let $h, \gamma \in[0,1], T, M, \mu \geq 0$. We say that a measurable function $f:(0, T] \times$ $\mathbb{R} \rightarrow \mathbb{R}$ is in the space $\mathcal{C}_{(0, T]}^{h}(\gamma, \mu, M)$ if

$$
|f(t, z)-f(s, z)| \leq M|t-s|^{h} s^{-\gamma}\left(|z|^{\mu} \vee 1\right), \quad 0<s<t \leq T, z \in \mathbb{R}
$$

and $|f(t, z)| \leq M\left(|z|^{\mu} \vee 1\right)$ for $z \in \mathbb{R}, t \in(0, T]$.

2) We say that a function $f:(0, T] \times \mathbb{R} \rightarrow \mathbb{R}$ is in the space $\mathcal{C}_{(0, T]}^{h-}(\gamma, 0+)$ if for any $\varepsilon>0$ there exists $M>0$ such that $f \in \mathcal{C}_{(0, T]}^{h-\varepsilon}(\gamma, \varepsilon, M)$.

If there is no ambiguity in time interval, we will frequently drop the subscript $(0, T]$ and write $\mathcal{C}^{h}$ instead of $\mathcal{C}_{(0, T]}^{h}$.

THEOREM 2.3. Let $b \in \mathbf{B}$. There exists a set $\Omega^{\prime \prime}=\Omega^{\prime \prime}(b) \subset \Omega$ with the following properties:

1) $\mathrm{P}\left(\Omega^{\prime \prime}\right)=1$.

2) Let $\omega \in \Omega^{\prime \prime}$. Then for any $0<\varepsilon<3 / 4, T>0, h \in(1 / 2,1], M>0$ there exists a constant $K_{b}=K_{b}(b, \omega, \varepsilon, T, M, h)<\infty$ such that for any $\gamma \in[0,1], \mu>0$, $f \in \mathcal{C}_{(0, T]}^{h}(\gamma, \mu, M), x, y, z \in \mathbb{R}, 0 \leq t_{1} \leq t_{2} \leq T, s \in[0, T]$ we have

$$
\begin{aligned}
& \left|\int_{t_{1}}^{t_{2}}(b(V(t+s, z)+f(t, z)+x)-b(V(t+s, z)+f(t, z)+y)) d t\right| \\
& \quad \leq K_{b}(\omega)|x-y|\left(t_{2}-t_{1}\right)^{1-\left(\frac{\gamma}{4 h-1} \vee \frac{1}{4}\right)-\varepsilon}(|x| \vee|y| \vee 1)^{1+\varepsilon}\left(|z|^{3 \mu+\varepsilon} \vee 1\right) .
\end{aligned}
$$

Furthermore, $\mathrm{E} K_{b} \leq\|b\|_{\infty} C(\varepsilon, T, M, h)$ for some function $C$ that does not depend on $b$. 
If the function $b$ were a Lipschitz function, then the left-hand side of (2.4) would be bounded by $\left|t_{1}-t_{2}\right||x-y|$. In our case, when $b$ is just a bounded function, the left-hand side of (2.4) is obviously bounded by $\left|t_{1}-t_{2}\right|$. Theorem 2.3 implies that one can trade the regularity in $t$ to gain the regularity in $x$. In particular, we see from the above theorem that $\mathrm{P}$-almost surely the function

$$
x \mapsto \int_{t_{1}}^{t_{2}} b(V(t, z)+x) d t, \quad x \in \mathbb{R},
$$

is Lipschitz in $x$. Moreover, we have very good local control on coefficients.

SKETCH OF THE PROOF OF THEOREM 2.3. The proof is based on an application of a suitable version of Kolmogorov continuity theorem to a corresponding moment bound. This is done in Section 3. The calculation of the moment bound turned out to be rather complicated and it involves a number of technical estimates. We do it thoroughly in Section 6 utilizing some ideas from [3].

REMARK 2.4. We would like to note that while the good set $\Omega^{\prime}$ in Theorems 2.1 and 2.2 can be chosen independently of the initial condition $q, \Omega^{\prime}$ as well as $\Omega^{\prime \prime}$ from Theorem 2.3 might still depend on the drift function $b$.

It is interesting to compare smoothing properties of operator (2.3) to the smoothing properties of a similar operator with a Brownian motion $B$ in place of $V$; see [8], Corollary 2.2 and also [5], Lemmas 3.1 and 3.2. We see that since $V$ in the time variable is less regular than the Brownian motion, Theorem 2.3 guarantees a better smoothing.

The function $f$ appears in (2.3) due to the presence of the drift in our main equation (2.2). Note that in the original Davie's paper [5] the smoothing is considered without the drift term (this corresponds to the case $f \equiv 0$ ). That was possible due to the use of the Girsanov transformation for eliminating the drift. In other words, the "good" set $\Omega$ " in [5] depends on the drift $f$ and the initial condition. Since we are aimed at establishing the flow property for (2.2), we have to prove path-by-path uniqueness simultaneously for all initial conditions; see the proof of Theorem 2.2(a). Thus we have to prove that smoothing in Theorem 2.3 occurs simultaneously for all drifts $f$ and this cannot be achieved with Girsanov's transformation.

The rest of the paper is devoted to the proofs of the main results and is organized as follows. In Section 3, we prove Theorem 2.3. The proof of Theorem 2.1 is rather large and is split into two parts for the convenience of the reader. Namely, in Section 4 we establish a number of auxiliary lemmas and present the main part of the proof in Section 5. Theorem 2.2 is also proved in Section 5. An important moment bound that is exploited for the proof of Theorem 2.3 is derived in Section 6 . A technical lemma that is applied to prove smoothing properties of the noise is established in Section 7. Finally, a number of technical estimates concerning the 
Gaussian kernel and related functions as well as the proof of a global version of the Kolmogorov continuity theorem are put in the Supplementary Material [2].

Convention on constants. Throughout the paper, $C$ denotes a positive constant whose value may change from line to line. $K$ denotes a random constant whose value might depend on $\omega \in \Omega$.

3. Proof of Theorem 2.3. We start proving the main results by presenting a proof of Theorem 2.3. First we give here a version of the Kolmogorov theorem on a noncompact set (global version) that will be extensively used in this and other proofs in the paper.

Define for $w=\left(w_{1}, w_{2}\right) \in \mathbb{R}^{2}, a=\left(a_{1}, a_{2}\right) \in(0,1]^{2}$ a weighted norm $d_{a}$

$$
d_{a}(w):=\left|w_{1}\right|^{a_{1}}+\left|w_{2}\right|^{a_{2}} .
$$

LEMMA 3.1 (Kolmogorov continuity theorem). Let $X(x, y), x \in \mathbb{R}, y \in \mathbb{R}^{2}$, be a continuous random field with values in $\mathbb{R}$. Assume that there exist nonnegative constants $a=\left(a_{1}, a_{2}\right) \in(0,1]^{2}, \alpha, \beta_{1}, \beta_{2}, C$ such that the inequalities

$$
\begin{aligned}
\mathrm{E} \mid X\left(x_{1}, y_{1}\right)-X\left(x_{1}, y_{2}\right)-X\left(x_{2}, y_{1}\right) & +\left.X\left(x_{2}, y_{2}\right)\right|^{\alpha} \\
& \leq C\left|x_{1}-x_{2}\right|^{\beta_{1}} d_{a}\left(y_{1}-y_{2}\right)^{\beta_{2}},
\end{aligned}
$$

$$
\mathrm{E}\left|X\left(x_{1}, y_{1}\right)-X\left(x_{2}, y_{1}\right)\right|^{\alpha} \leq C\left|x_{1}-x_{2}\right|^{\beta_{1}}
$$

hold for any $x_{1}, x_{2} \in \mathbb{R}, y_{1}, y_{2} \in \mathbb{R}^{2},\left|x_{1}-x_{2}\right| \leq 1,\left|y_{1}-y_{2}\right| \leq 1$.

Then for any $\gamma_{1} \in\left(0,\left(\beta_{1}-1\right) / \alpha\right)$ and $\gamma_{2} \in\left(0,\left(\beta_{2}-1 / a_{1}-1 / a_{2}\right) / \alpha\right)$ there exist a set $\Omega^{*} \subset \Omega$ with $\mathrm{P}\left(\Omega^{*}\right)=1$ and a random variable $K$ with $\mathrm{E} K(\omega)^{\alpha} \leq C_{1}$ such that for any $\omega \in \Omega^{\prime}, x_{1}, x_{2} \in \mathbb{R}, y_{1}, y_{2} \in \mathbb{R}^{2},\left|x_{1}-x_{2}\right| \leq 1,\left|y_{1}-y_{2}\right| \leq 1$ we have

$$
\begin{aligned}
& \left|X\left(x_{1}, y_{1}\right)-X\left(x_{1}, y_{2}\right)-X\left(x_{2}, y_{1}\right)+X\left(x_{2}, y_{2}\right)\right| \\
& \quad \leq K(\omega)\left(\left|x_{1}\right| \vee\left|y_{1}\right| \vee 1\right)^{3 / \alpha}\left|x_{1}-x_{2}\right|^{\gamma_{1}} d_{a}\left(y_{1}-y_{2}\right)^{\gamma_{2}}
\end{aligned}
$$

and

$$
\left|X\left(x_{1}, y_{1}\right)-X\left(x_{2}, y_{1}\right)\right| \leq K(\omega)\left(\left|x_{1}\right| \vee\left|y_{1}\right| \vee 1\right)^{3 / \alpha}\left|x_{1}-x_{2}\right|^{\gamma_{1}},
$$

where the constant $C_{1}>0$ depends on the field $X$ only through a, $\alpha, \beta_{i}, \gamma_{i}, C$.

The proof of the lemma uses a local version of the Kolmogorov continuity theorem (see, e.g., [18], Theorem 1.4.4, [15], Theorem 3.1 and [24], Corollary 1.2) and is given in the Supplementary Material [2], Section 1.

The proof of Theorem 2.3 is based on the above mentioned version of the Kolmogorov theorem and the following moment bound. 
Proposition 3.2. Let $b: \mathbb{R} \rightarrow \mathbb{R}$ be a bounded differentiable function with bounded derivative. Then for any $0 \leq t_{1} \leq t_{2} \leq T, z, z_{1}, z_{2}, x, y \in \mathbb{R},\left|z_{1}-z_{2}\right| \leq 1$, $\delta \in(0,1), \delta^{\prime} \in(0, \delta), p>1$ we have

$$
\begin{aligned}
& \mathrm{E}\left|\int_{t_{1}}^{t_{2}}\left(b^{\prime}\left(V\left(t, z_{1}\right)+x\right)-b^{\prime}\left(V\left(t, z_{2}\right)+y\right)\right) d t\right|^{p} \\
& \leq C\left(t_{2}-t_{1}\right)^{p(3 / 4-\delta / 4)}\left(\left|z_{1}-z_{2}\right|^{p \delta^{\prime} / 2}+|x-y|^{p \delta}\right) ; \\
& \mathrm{E}\left|\int_{t_{1}}^{t_{2}} b^{\prime}(V(t, z)) d t\right|^{p} \leq C\left(t_{2}-t_{1}\right)^{p(3 / 4-\delta)} .
\end{aligned}
$$

for some constant $C=C\left(p, T, \delta, \delta^{\prime},\|b\|_{\infty}\right)>0$.

It is important to stress that the constant $C$ from Proposition 3.2 depends only on $\|b\|_{\infty}$ but not on the function $b$ itself and not on its derivative. The proof of Proposition 3.2 is postponed to Section 6.

Finally, we need a technical estimate.

LEMMA 3.3. Let $U \subset \mathbb{R}$ and assume that $U$ has a Lebesgue measure 0 . Then there exists a set $\Omega(U) \subset \Omega$ such that $\mathrm{P}(\Omega(U))=1$ and for any $\omega \in \Omega(U), h>$ $1 / 2, T>0, M>0, \mu>0, f \in \mathcal{C}_{(0, T]}^{h}(1, \mu, M), z \in \mathbb{R}, s \in[0, T]$ we have

$$
\int_{0}^{T} \mathbb{1}_{U}(V(t+s, z, \omega)+f(t, z)) d t=0 .
$$

This lemma is proved in Section 7.

Proof of THE TheOREM 2.3. First we consider the case when $b$ is a bounded differentiable function with a continuous bounded derivative and $\|b\|_{\infty}=1$. In this case, we apply a version of the Kolmogorov continuity theorem (Lemma 3.1) to the random field

$$
X(t,(z, x)):=\int_{0}^{t} b^{\prime}(V(r, z)+x) d r .
$$

Fix arbitrary $T>0$. It follows from Proposition 3.2 and Lemma 3.1 that for any $\delta \in(0,1), \varepsilon>0$ there exist a set $\Omega_{T, \delta, \varepsilon} \subset \Omega$ with $\mathrm{P}\left(\Omega_{T, \delta, \varepsilon}\right)=1$ and a random variable $K(\omega)$ such that for all $z_{1}, z_{2}, x, y \in \mathbb{R}$ with $\left|z_{1}-z_{2}\right|+|x-y| \leq 1$ and $0 \leq t_{1} \leq t_{2} \leq 2 T, \omega \in \Omega_{T, \delta, \varepsilon}$ one has

$$
\begin{aligned}
\mid \int_{t_{1}}^{t_{2}}( & \left.b^{\prime}\left(V\left(t, z_{1}\right)+x\right)-b^{\prime}\left(V\left(t, z_{2}\right)+y\right)\right) d t \mid \\
\leq & K(\omega)\left(t_{2}-t_{1}\right)^{(3 / 4-\delta / 4-\varepsilon)}\left(\left|z_{1}-z_{2}\right|^{\delta / 2}+|x-y|^{\delta}\right)^{1-\varepsilon} \\
& \times\left(|x|^{\varepsilon} \vee|y|^{\varepsilon} \vee 1\right)\left(\left|z_{1}\right|^{\varepsilon} \vee\left|z_{2}\right|^{\varepsilon} \vee 1\right)
\end{aligned}
$$


and $\mathrm{E} K \leq C$, where the constant $C=C(T, \delta, \varepsilon)$ does not depend on the function $b$.

We apply now the above inequality to $z_{1}=z_{2}=z$ and arbitrary $x, y \in \mathbb{R}$. That is, if $|x-y| \leq N$ we apply the above inequality $N$ times. We get that on $\Omega_{T, \delta, \varepsilon}$ for all $x, y, z \in \mathbb{R}, 0 \leq t_{1} \leq t_{2} \leq T, s \in[0, T]$,

$$
\begin{aligned}
& \left|\int_{t_{1}}^{t_{2}}\left(b^{\prime}(V(t+s, z)+x)-b^{\prime}(V(t+s, z)+y)\right) d t\right| \\
& \quad \leq K_{1}(\omega)\left(|x|^{1+\varepsilon} \vee|y|^{1+\varepsilon} \vee 1\right)\left(|z|^{\varepsilon} \vee 1\right)\left(t_{2}-t_{1}\right)^{3 / 4-\delta / 4-\varepsilon}|x-y|^{\delta(1-\varepsilon)},
\end{aligned}
$$

where we have also applied change of variables $t \rightarrow t+s$ in the integral. Here $\mathrm{E} K_{1} \leq C=C(T, \delta, \varepsilon)$.

In a similar way, inequality (3.4) and Proposition 3.2 yield that for any $\varepsilon \in(0,3 / 4)$ there exists a set $\widetilde{\Omega}_{T, \varepsilon}$ and a random variable $K_{2}(\omega)$ such that for any $\omega \in \widetilde{\Omega}_{T, \mathcal{E}}, z \in \mathbb{R}, 0 \leq t_{1} \leq t_{2} \leq T, s \in[0, T]$ we have

$$
\left|\int_{t_{1}}^{t_{2}} b^{\prime}(V(t+s, z)) d t\right| \leq K_{2}(\omega)\left(|z|^{\varepsilon} \vee 1\right)\left(t_{2}-t_{1}\right)^{3 / 4-\varepsilon} .
$$

Again, $\mathrm{E} K_{2} \leq C=C(T, \varepsilon)$.

This allows us to proceed to the next step. Fix $M>0$ and take any function $f \in \mathcal{C}_{(0, T]}^{h}(\gamma, \mu, M)$. Fix $0 \leq t_{1} \leq t_{2} \leq T$. Consider the following binary partition of the interval $\left[t_{1}, t_{2}\right]$ :

$$
t_{n}^{i}:=t_{1}+\left(t_{2}-t_{1}\right) i 2^{-n}, \quad n \in \mathbb{Z}_{+}, i=0,1, \ldots, 2^{n} .
$$

Let $f_{n}$ be the following piecewise-constant approximation of $f$ :

$$
f_{n}(t, z):=\sum_{i=0}^{2^{n}-1} \mathbb{1}\left(t \in\left(t_{n}^{i}, t_{n}^{i+1}\right]\right) f\left(t_{n}^{i+1}, z\right), \quad t \in\left[t_{1}, t_{2}\right], z \in \mathbb{R} .
$$

Clearly, the sequence of functions $f_{n}$ converges pointwise to $f$ on $\left[t_{1}, t_{2}\right]$. Thus, for arbitrary $s \in[0, T], \omega \in \Omega, x, y, z \in \mathbb{R}$ we derive

$$
\begin{aligned}
& \left|\int_{t_{1}}^{t_{2}}(b(V(t+s, z)+f(t, z)+x)-b(V(t+s, z)+f(t, z)+y)) d t\right| \\
& \quad=\left|\int_{x}^{y} \int_{t_{1}}^{t_{2}} b^{\prime}(V(t+s, z)+f(t, z)+r) d t d r\right| \\
& \quad \leq\left|\int_{x}^{y} \int_{t_{1}}^{t_{2}} b^{\prime}\left(V(t+s, z)+f_{0}(t, z)+r\right) d t d r\right|+\sum_{k=0}^{\infty} J\left(k, t_{1}, t_{2}\right) \\
& \quad=: I+\sum_{k=0}^{\infty} J\left(k, t_{1}, t_{2}\right),
\end{aligned}
$$


where in the first identity we have used Fubini's theorem (recall that we have assumed boundedness of the function $\left.b^{\prime}\right)$ and denoted for $k \in \mathbb{Z}_{+}$and $l_{1}, l_{2} \in\left[t_{1}, t_{2}\right]$,

$$
\begin{aligned}
& J\left(k, l_{1}, l_{2}\right) \\
& :=\left|\int_{x}^{y} \int_{l_{1}}^{l_{2}}\left(b^{\prime}\left(V(t+s, z)+f_{k+1}(t, z)+r\right)-b^{\prime}\left(V(t+s, z)+f_{k}(t, z)+r\right)\right) d t d r\right| .
\end{aligned}
$$

It turns out that we need to apply (3.7) with different $\delta$ to estimate $I$ and $J\left(k, t_{1}, t_{2}\right)$. Since, by definition, the function $f_{0}$ is constant on $\left[t_{1}, t_{2}\right]$, we see that (3.7) with $\delta=\varepsilon$ together with (3.8) yield for $\omega \in \widetilde{\Omega}_{T, \varepsilon} \cap \Omega_{T, \varepsilon, \varepsilon}$

$$
\begin{aligned}
I \leq & \left|\int_{x}^{y} \int_{t_{1}}^{t_{2}} b^{\prime}\left(V(t+s, z)+f_{0}(t, z)+r\right)-b^{\prime}(V(t+s, z)) d t d r\right| \\
& +\left|\int_{x}^{y} \int_{t_{1}}^{t_{2}} b^{\prime}(V(t+s, z)) d t d r\right| \\
\leq & K_{3}(\omega)(|x| \vee|y| \vee 1)^{1+2 \varepsilon}\left(|z|^{2 \mu+\varepsilon} \vee 1\right)\left(t_{2}-t_{1}\right)^{3 / 4-2 \varepsilon}|x-y|
\end{aligned}
$$

and $\mathrm{E} K_{3} \leq C(T, \varepsilon, M)$.

Recall that each function $f_{k}$ is a piecewise constant function in $t$. Therefore, to estimate $J\left(k, t_{1}, t_{2}\right)$ we split the integral over $\left[t_{1}, t_{2}\right]$ into integrals over intervals $\left(t_{k}^{i}, t_{k+1}^{2 i+1}\right], i=0,1, \ldots, 2^{k}-1$, where $f_{k}$ and $f_{k+1}$ are constant in $t$, and apply estimate (3.7) to each of these integrals. Note that $f_{k}=f_{k+1}$ on the complement of the union of these intervals. Thus, for any $k \in \mathbb{Z}_{+}, i=0,1, \ldots, 2^{k}-1$ we obtain on $\Omega_{T, \delta, \varepsilon}$

$$
\begin{aligned}
& J\left(k, t_{k}^{i}, t_{k}^{i+1}\right) \\
& \leq J\left(k, t_{k}^{i}, t_{k+1}^{2 i+1}\right)+J\left(k, t_{k+1}^{2 i+1}, t_{k}^{i+1}\right) \\
&=\left|\int_{x}^{y} \int_{t_{k}^{i}}^{t_{k+1}^{2 i+1}}\left(b^{\prime}\left(V(t+s, z)+f\left(t_{k+1}^{2 i+1}, z\right)+r\right)-b^{\prime}\left(V(t+s, z)+f\left(t_{k}^{i+1}, z\right)+r\right)\right) d t d r\right| \\
&+\left|\int_{x}^{y} \int_{t_{k+1}^{t_{k}}}^{t^{i+1}}\left(b^{\prime}\left(V(t+s, z)+f\left(t_{k}^{i+1}, z\right)+r\right)-b^{\prime}\left(V(t+s, z)+f\left(t_{k}^{i+1}, z\right)+r\right)\right) d t d r\right| \\
& \leq C K_{1}(\omega)(|x| \vee|y| \vee 1)^{1+\varepsilon}\left(|z|^{2 \mu+\varepsilon} \vee 1\right)\left(t_{2}-t_{1}\right)^{3 / 4-\delta / 4-\varepsilon} \\
&\left.\times 2^{-(3 / 4-\delta / 4-\varepsilon) k} \mid f\left(t_{k+1}^{2 i+1}, z\right)-f_{k}^{i+1}, z\right)\left.\right|^{\delta-\varepsilon}|x-y| \\
& \leq C K_{1}(\omega)(|x| \vee|y| \vee 1)^{1+\varepsilon}\left(|z|^{3 \mu+\varepsilon} \vee 1\right)\left(t_{2}-t_{1}\right)^{3 / 4+\delta(h-1 / 4-\gamma)-2 \varepsilon} \\
& \times 2^{-k(3 / 4-\delta(1 / 4-h+\gamma)-3 \varepsilon)}(i+1 / 2)^{-\gamma \delta}|x-y|,
\end{aligned}
$$

where in the last line we used the fact that $f \in \mathcal{C}_{(0, T]}^{h}(\gamma, \mu, M)$ and the constant $C=C(T, \varepsilon, M)$ does not depend on $i$ and $k$. By summing the obtained inequality 
over all $k \in \mathbb{Z}_{+}$and $i \in\left[0,2^{k}-1\right]$, we deduce on $\Omega_{T, \delta, \varepsilon}$

$$
\begin{aligned}
\sum_{k=0}^{\infty} J\left(k, t_{1}, t_{2}\right)= & \sum_{k=0}^{\infty} \sum_{i=0}^{2^{k}-1} J\left(k, t_{k}^{i}, t_{k}^{i+1}\right) \\
\leq & C K_{1}(\omega)(|x| \vee|y| \vee 1)^{1+\varepsilon}\left(|z|^{3 \mu+\varepsilon} \vee 1\right) \\
& \times\left(t_{2}-t_{1}\right)^{3 / 4+\delta(h-1 / 4-\gamma)-2 \varepsilon}|x-y| \\
& \times \sum_{k=0}^{\infty} 2^{k(1 / 4-\delta(h-1 / 4)+3 \varepsilon)}
\end{aligned}
$$

Again, $C=C(T, \delta, \varepsilon, M)$. We see that in order for the sum in the right-hand side of (3.11) to be convergent we must necessarily have

$$
\delta(h-1 / 4)>1 / 4+3 \varepsilon .
$$

We must also have $\delta<1$. Recall that by assumption of the theorem $h>1 / 2$. Thus one can take $\delta:=1 /(4 h-1)+24 \varepsilon$. If, additionally, $\gamma \leq h-1 / 4$, then combining (3.9), (3.10), (3.11), we finally obtain for $\varepsilon>0$ on $\bar{\Omega}_{T, \varepsilon, h}^{*}:=\widetilde{\Omega}_{T, \varepsilon} \cap \Omega_{T, \varepsilon, \varepsilon} \cap$ $\Omega_{T, 1 /(4 h-1)+24 \varepsilon, \varepsilon}$

$$
\begin{gathered}
\left|\int_{t_{1}}^{t_{2}}(b(V(t+s, z)+f(t, z)+x)-b(V(t+s, z)+f(t, z)+y)) d t\right| \\
\quad \leq K_{4}(\omega)|x-y|(|x| \vee|y| \vee 1)^{1+2 \varepsilon}\left(|z|^{3 \mu+\varepsilon} \vee 1\right)\left(t_{2}-t_{1}\right)^{3 / 4-2 \varepsilon}
\end{gathered}
$$

In case $\gamma>h-1 / 4$, we obtain on $\Omega_{T, \varepsilon, h}^{*}$ :

$$
\begin{aligned}
& \left|\int_{t_{1}}^{t_{2}}(b(V(t+s, z)+f(t, z)+x)-b(V(t+s, z)+f(t, z)+y)) d t\right| \\
& \quad \leq K_{4}(\omega)|x-y|(|x| \vee|y| \vee 1)^{1+2 \varepsilon}\left(|z|^{3 \mu+\varepsilon} \vee 1\right)\left(t_{2}-t_{1}\right)^{1-\gamma /(4 h-1)-24 \varepsilon} .
\end{aligned}
$$

Note that in both cases we have $\mathrm{E} K_{4}(\omega) \leq C(T, \varepsilon, M, h)$. Now we set $\Omega^{*}:=$ $\cap \Omega_{T, \varepsilon, h}^{*}$ where the intersection is taken over all rational $T>0, \varepsilon>0, h \in$ $(1 / 2,1]$. We see that on $\Omega^{*}$ the statement of the theorem holds. This concludes the proof of the theorem for the case where $b$ is a bounded differentiable function with a continuous bounded derivative and $\|b\|_{\infty}=1$.

If $\|b\|_{\infty}=0$, then there is nothing to prove. If $b$ is a bounded differentiable function with a continuous bounded derivative but $\|b\|_{\infty} \neq 1,\|b\|_{\infty}>0$, then the statement of the theorem also holds. Indeed, we can renormalize $b$ and consider $b_{1}(x):=b(x) /\|b\|_{\infty}$.

Finally, to prove the theorem in the general case (for bounded but not necessarily differentiable $b$ with arbitrarily $\|b\|_{\infty}$ ) we use approximations. It follows from Lusin's theorem that there exists a sequence $\left(b_{n}\right)_{n \in \mathbb{Z}_{+}}$of bounded differentiable function with continuous bounded derivatives such that

$$
\lim _{n \rightarrow \infty} b_{n}(x)=b(x) \quad \text { Lebesgue-almost everywhere in } x ; x \in \mathbb{R}
$$


and $\sup _{n}\left\|b_{n}\right\|_{\infty} \leq 2\|b\|_{\infty}$. Put $U:=\left\{x \in \mathbb{R}: \lim _{n \rightarrow \infty} b_{n}(x) \neq b(x)\right\}, \tilde{b}(x):=$ $\lim _{n \rightarrow \infty} b_{n}(x)$. We see that the set $U$ is of Lebesgue measure 0 .

Let $\Omega_{n}$ be the "good" set for the function $b_{n}$ (i.e., the set such that the statement of the theorem is satisfied for the function $\left.b_{n}\right)$. By above, $\mathrm{P}\left(\Omega_{n}\right)=1$. Take

$$
\Omega_{\infty}:=\bigcap_{n=1}^{\infty} \Omega_{n} \cap \Omega(U),
$$

where the set $\Omega(U)$ is defined in Lemma 3.3. Clearly, $\mathrm{P}\left(\Omega_{\infty}\right)=1$. Take arbitrary $T>0, M>0, h>1 / 2,0<\varepsilon<3 / 4$. By the dominated convergence theorem and Lemma 3.3, we have on $\Omega_{\infty}$ for any $\gamma \in[0,1], \mu>0, f \in \mathcal{C}_{(0, T]}^{h}(\gamma, \mu, M)$, $x, y, z \in \mathbb{R}, 0 \leq t_{1} \leq t_{2} \leq T, s \in[0, T]$

$$
\begin{aligned}
& \left|\int_{t_{1}}^{t_{2}}(b(V(t+s, z)+f(t, z)+x)-b(V(t+s, z)+f(t, z)+y)) d t\right| \\
& \leq\left|\int_{t_{1}}^{t_{2}}(\tilde{b}(V(t+s, z)+f(t, z)+x)-\widetilde{b}(V(t+s, z)+f(t, z)+y)) d t\right| \\
& \quad+3 \int_{t_{1}}^{t_{2}} \mathbb{1}_{U}(V(t+s, z)+f(t, z)+x) d t \\
& \quad+3 \int_{t_{1}}^{t_{2}} \mathbb{1}_{U}(V(t+s, z)+f(t, z)+y) d t \\
& =\liminf _{n \rightarrow \infty}\left|\int_{t_{1}}^{t_{2}}\left(b_{n}(V(t+s, z)+f(t, z)+x)-b_{n}(V(t+s, z)+f(t, z)+y)\right) d t\right| \\
& \leq|x-y|(|x| \vee|y| \vee 1)^{1+\varepsilon}\left(|z|^{3 \mu+\varepsilon} \vee 1\right)\left(t_{2}-t_{1}\right)^{1-1 / 4\left(\frac{\gamma}{h-1 / 4} \vee 1\right)-\varepsilon} \liminf _{n \rightarrow \infty} K_{b_{n}}(\omega),
\end{aligned}
$$

where $K_{b_{n}}$ is the corresponding constant from Theorem 2.3. For $\omega \in \Omega_{\infty}$, put $K_{b}(\omega):=\liminf _{n \rightarrow \infty} K_{b_{n}}(\omega)$. By Fatou's lemma,

$$
\mathrm{E} K_{b}(\omega) \leq \liminf _{n \rightarrow \infty} \mathrm{E} K_{b_{n}}(\omega) \leq C(\varepsilon, T, M, h) \sup _{n}\left\|b_{n}\right\|_{\infty} .
$$

Thus the random variable $K_{b}(\omega)$ has a finite expectation. Hence there exists a set $\Omega^{\prime \prime} \subset \Omega_{\infty}$ such that $\mathrm{P}\left(\Omega^{\prime \prime}\right)=1$ and on $\Omega^{\prime \prime}$ we have $K_{b}(\omega)<\infty$. This together with the above estimate concludes the proof of the theorem.

4. Preparation steps for proving Theorem 2.1. In this section, we prepare for the proof of our main result, that is, Theorem 2.1. In particular, we will select a specific "good" set $\Omega^{\prime}$ of full probability measure and in the next section we will prove that equation (2.2) indeed has a unique solution on $\Omega^{\prime}$.

First we need to introduce approximation operator in the following way. Let $f:[0,1] \rightarrow \mathbb{R}$ be a continuous function. We define a piecewise-constant approximation of $f$ as follows. For $n \in \mathbb{Z}_{+}$, put

$$
\lambda_{n}(f)(t):=\sum_{i=0}^{2^{n}-1} \mathbb{1}_{\left(i 2^{-n},(i+1) 2^{-n}\right]}(t) f\left((i+1) 2^{-n}\right) .
$$


In other words, $\lambda_{n}(f)$ is a piecewise-constant function that takes constant values on intervals of length $2^{-n}$.

Many times in the proofs of the theorems it will be convenient to work with a shifted solution to (2.2). Thus we define

$$
u_{s, q}^{*}(t, \cdot):=u_{s, q}(t+s, \cdot), \quad t \geq 0,
$$

where we recall that $u_{s, q}$ stands for the solution to (2.2) that starts from the initial condition $q$ at times $s$. It is easy to see that $u_{s, q}^{*}$ satisfies the following equation for any $t>0, z \in \mathbb{R}$ :

$$
\begin{aligned}
u_{s, q}^{*}(t, z, \omega)= & \int_{\mathbb{R}} p_{t}\left(z-z^{\prime}\right) q\left(z^{\prime}, \omega\right) d z^{\prime} \\
& +\int_{0}^{t} \int_{\mathbb{R}} p_{t-t^{\prime}}\left(z-z^{\prime}\right) b\left(u_{s, q}^{*}\left(t^{\prime}, z^{\prime}, \omega\right)\right) d z^{\prime} d t^{\prime}+V(s, t+s, z, \omega), \\
u_{s, q}^{*}(0, z, \omega)= & q(z, \omega) .
\end{aligned}
$$

REMARK 4.1. Clearly, equation (2.2) has a unique solution if and only if equation (4.3) has a unique solution.

We introduce also the notation for the difference between two Gaussian kernels by setting

$$
\Delta p_{t}\left(z_{1}, z_{2}\right):=p_{t}\left(z_{1}\right)-p_{t}\left(z_{2}\right), \quad t>0, z_{1}, z_{2} \in \mathbb{R} .
$$

Further, we will need to consider weighted norms. So for $\delta \geq 0$ we define weight function

$$
\Lambda_{\delta}(x):=e^{x} x^{\delta}, \quad x \geq 0 .
$$

Consider also a class of globally Lipschitz functions.

Definition 4.1. We say that a function $f \in \mathbf{B}$ belongs to the class $\mathcal{C}_{\text {Lip }}^{b}$, if there exists a constant $C>0$ such that $\left|f\left(z_{1}\right)-f\left(z_{2}\right)\right| \leq C\left|z_{1}-z_{2}\right|$ for any $z_{1}, z_{2} \in \mathbb{R}$.

Finally, we will also need the following process:

$$
\mathcal{V}(r, s, t, z):=\int_{r}^{s} \int_{\mathbb{R}} p_{t-t^{\prime}}\left(z-z^{\prime}\right) W\left(d t^{\prime}, d z^{\prime}\right), \quad 0 \leq r \leq s \leq t, z \in \mathbb{R} .
$$

We see that, by definition, $V(s, t, z)=\mathcal{V}(s, t, t, z)$. 
4.1. Estimates involving Gaussian density. First let us give a number of very simple lemmas involving the Gaussian kernel. Their proofs are standard; for the sake of completeness, we provide their proofs in the Supplementary Material [2], Section 2.

LEMMA 4.2. For any $\delta_{1}, \delta_{2} \in[0,1]$, there exists $C=C\left(\delta_{1}, \delta_{2}\right)$ such that for any $a_{1}, a_{2} \in \mathbb{R}, t>0$ we have the following bounds:

$$
\begin{aligned}
& \int_{\mathbb{R}}\left|p_{t}\left(x+a_{1}\right)-p_{t}(x)\right| d x \leq C\left|a_{1}\right|^{\delta_{1}} t^{-\delta_{1} / 2} \\
& \int_{\mathbb{R}}\left|\frac{\partial p_{t}}{\partial x}\left(x+a_{1}\right)-\frac{\partial p_{t}}{\partial x}(x)\right| d x \leq C\left|a_{1}\right|^{\delta_{1}} t^{-\left(1+\delta_{1}\right) / 2} ; \\
& \int_{\mathbb{R}}\left|\frac{\partial p_{t}}{\partial x}\left(x+a_{1}+a_{2}\right)-\frac{\partial p_{t}}{\partial x}\left(x+a_{1}\right)-\frac{\partial p_{t}}{\partial x}\left(x+a_{2}\right)+\frac{\partial p_{t}}{\partial x}(x)\right| d x \\
& \leq C\left|a_{1}\right|^{\delta_{1}}\left|a_{2}\right|^{\delta_{2}} t^{-\left(1+\delta_{1}+\delta_{2}\right) / 2}
\end{aligned}
$$

LEMMA 4.3. For any $T>0, \delta \geq 0$, there exists $C=C(T, \delta)>0$ such that for any $s, t \in[0, T]$, we have

$$
\int_{\mathbb{R}}\left|p_{t}(z)-p_{s}(z)\right|\left(|z|^{\delta} \vee 1\right) d z \leq C|\log t-\log s| .
$$

LEMMA 4.4. For any $\delta \in(0,1 / 6), T>0$, there exists $C=C(T, \delta)>0$ such that for any $0<t_{1}<t_{2}<t, z_{1}, z_{2}, z \in \mathbb{R}$ we have

$\int_{\mathbb{R}} p_{t}\left(z-z^{\prime}\right) \Lambda_{\delta}\left(\left|z^{\prime}\right| \vee 1\right) d z^{\prime} \leq C \Lambda_{\delta}(|z| \vee 1)$

4.11) $\int_{\mathbb{R}} \int_{t_{1}}^{t_{2}}\left|\frac{\partial}{\partial t^{\prime}} p_{t-t^{\prime}}\left(z-z^{\prime}\right)\right|\left(t_{2}-t^{\prime}\right)^{2 / 3-\delta} \Lambda_{\delta}\left(\left|z^{\prime}\right| \vee 1\right) d t^{\prime} d z^{\prime}$

$$
\leq C\left|t_{2}-t_{1}\right|^{2 / 3-\delta} \Lambda_{\delta}(|z| \vee 1)
$$

$$
\begin{gathered}
\int_{\mathbb{R}}\left|p_{t}\left(z_{1}-z^{\prime}\right)-p_{t}\left(z_{2}-z^{\prime}\right)\right| \Lambda_{\delta}\left(\left|z^{\prime}\right| \vee 1\right) d z^{\prime} \\
\leq C t^{-1 / 2}\left|z_{1}-z_{2}\right| \Lambda_{\delta}\left(\left|z_{1}\right| \vee\left|z_{2}\right| \vee 1\right)
\end{gathered}
$$

$$
\begin{gathered}
\int_{\mathbb{R}} \int_{t_{1}}^{t_{2}}\left|\frac{\partial}{\partial t^{\prime}}\left(p_{t-t^{\prime}}\left(z_{1}-z^{\prime}\right)-p_{t-t^{\prime}}\left(z_{2}-z^{\prime}\right)\right)\right|\left(t_{2}-t^{\prime}\right)^{2 / 3-\delta} \Lambda_{\delta}\left(\left|z^{\prime}\right| \vee 1\right) d t^{\prime} d z^{\prime} \\
\leq C\left(t_{2}-t_{1}\right)^{2 / 3-\delta}\left(t-t_{1}\right)^{-1 / 2}\left|z_{1}-z_{2}\right| \Lambda_{\delta}\left(\left|z_{1}\right| \vee\left|z_{2}\right| \vee 1\right)
\end{gathered}
$$

LEMMA 4.5. Let $f:[0, \infty) \times \mathbb{R} \rightarrow \mathbb{R}$ be a bounded measurable function. Define

$$
h(t, z):=\int_{0}^{t} \int_{\mathbb{R}} p_{t-t^{\prime}}\left(z-z^{\prime}\right) f\left(t^{\prime}, z^{\prime}\right) d z^{\prime} d t^{\prime}, \quad z \in \mathbb{R}, t \geq 0 .
$$


Then for any $T>0, \delta>0$, there exists a constant $C=C(T, \delta)$ such that for any $t_{1}, t_{2} \in[0, T], z_{1}, z_{2} \in \mathbb{R}$ we have

$$
\left|h\left(t_{1}, z_{1}\right)-h\left(t_{2}, z_{2}\right)\right| \leq C\|f\|_{\infty}\left(\left|z_{1}-z_{2}\right|+\left|t_{1}-t_{2}\right|^{1-\delta}\right) .
$$

Lemma 4.6. Let $\mu \geq 0$ and let $q \in \mathbf{B}(\mu)$, that is, for some $M>0$ we have $|q(z)| \leq M\left(|z|^{\mu} \vee 1\right), z \in \mathbb{R}$. Then for any $T>0$ there exists a constant $C=$ $C(T, \mu)$ such that function

$$
h(t, z):=\int_{\mathbb{R}} p_{t}\left(z-z^{\prime}\right) q\left(z^{\prime}\right) d z^{\prime}, \quad z \in \mathbb{R}, t \in[0, T]
$$

belongs to the class $\mathcal{C}_{(0, T]}^{1}(1, \mu, C M)$. The constant $C$ does not depend on the function $q$.

Further, if $q \in \mathcal{C}_{\text {Lip }}^{b}$, then there exists a constant $C_{1}=C_{1}(T, q)$ such that for any $t_{1}, t_{2} \in[0, T], z_{1}, z_{2} \in \mathbb{R}$ we have

$$
\left|h\left(t_{1}, z_{1}\right)-h\left(t_{2}, z_{2}\right)\right| \leq C_{1}\left(\left|z_{1}-z_{2}\right|+\left|t_{1}-t_{2}\right|^{1 / 2}\right) .
$$

4.2. Existence of a regular version of $V$ and its properties. The next lemma establishes the global regularity properties of the noise process $\mathcal{V}$ (recall the definition of $\mathcal{V}$ given in (4.6)). The proof of the lemma is technical and follows the usual line of argument. We provide it in the Supplementary Material [2], Section 3.

LEMma 4.7. There exists a set $\Omega_{V} \subset \Omega$ with the following properties:

1) $\mathrm{P}\left(\Omega_{V}\right)=1$.

2) Let $\omega \in \Omega_{V}$. Then the functions $(s, t, z) \mapsto V(s, t, z, \omega)$ and $(r, s, t, z) \mapsto$ $\mathcal{V}(r, s, t, z, \omega)$ are continuous. Furthermore, for any $T>0, \varepsilon \in(0,1 / 2), p>0$ there exists $K(\omega)=K(\omega, p, T, \varepsilon)$ such that for any $0 \leq s \leq t \leq T, 0 \leq s<$ $t_{1}<t_{2} \leq T, z, z_{1}, z_{2} \in \mathbb{R}$ we have

$$
\begin{aligned}
& \quad\left|\mathcal{V}\left(0, s, t, z_{1}\right)-\mathcal{V}\left(0, s, t, z_{2}\right)\right| \\
& \leq K(\omega)\left|z_{1}-z_{2}\right|^{1 / 2-\varepsilon}\left(\left|z_{1}\right|^{2 \varepsilon} \vee\left|z_{2}\right|^{2 \varepsilon} \vee 1\right) ; \\
& \text { 4.16) } \quad\left|\mathcal{V}\left(0, s, t_{1}, z\right)-\mathcal{V}\left(0, s, t_{2}, z\right)\right| \leq K(\omega)\left|t_{1}-t_{2}\right|\left(t_{1}-s\right)^{-1}\left(|z|^{\varepsilon}\right) \\
& \text { Moreover, } \omega \mapsto K(\omega) \text { is a random variable with } \mathrm{E} K(\omega)^{p}<\infty .
\end{aligned}
$$

Let us emphasize that the result is of course not surprising: it is well known that the convolution of the white noise with the heat kernel is locally Hölder $(1 / 2-\varepsilon)$ continuous in space and Hölder $(1 / 4-\varepsilon)$ continuous in time (see, e.g., [16], Exercise 6.9). The lemma gives uniform global control on Hölder coefficients. As one 
can expect, the price to pay is that Hölder coefficients are no longer bounded but grow as $|z|^{\varepsilon}$ if $z \rightarrow \infty$ (in other words, the convolution of the white noise with the heat kernel is not a globally Hölder function).

The next lemma provides some useful simple bounds for the variance of $\mathcal{V}$. Its proof is very standard and straightforward; thus we also put it in the Supplementary Material [2], Section 3.

LEMMA 4.8. For any $\delta \in[0,1]$, there exists a constant $C=C(\delta)>0$ such that for any $0 \leq r<s \leq t, z, z_{1}, z_{2} \in \mathbb{R}$ we have

(4.18) $\operatorname{Var} \mathcal{V}(r, s, t, z) \leq C(s-r)(t-r)^{-1 / 2}$;

(4.19) $\operatorname{Var}\left(\mathcal{V}\left(r, s, t, z_{1}\right)-\mathcal{V}\left(r, s, t, z_{2}\right)\right) \leq C\left|z_{1}-z_{2}\right|^{\delta}(s-r)(t-s)^{-1 / 2-\delta / 2}$;

(4.20) $\operatorname{Var}\left(\mathcal{V}\left(r, s, t, z_{1}\right)-\mathcal{V}\left(r, s, t, z_{2}\right)\right) \leq C\left|z_{1}-z_{2}\right|$.

4.3. Continuity lemmas. As we mentioned before, in order to prove Theorem 2.1 we approximate the drift in equation (2.2) by a sequence of piecewise continuous functions and pass to the limit (see the proof of Lemma 5.9 below). If the function $b$ were continuous, this would not require any additional clarifications. However, in our case, when we assume that $b$ is just a measurable bounded function we need to explain why the passage to the limit is justified here.

Recall the definition of the approximation operator $\lambda_{n}$ given in (4.1).

LEMMA 4.9. For any $\varepsilon>0, M>0, h>1 / 2, N \in \mathbb{N}, T>0, \mu>0$, there exists $\delta>0$ such that for each open set $U \subset \mathbb{R}$ with $|U| \leq \delta$ we have with probability greater or equal than $1-\varepsilon$

$$
\int_{0}^{T} \mathbb{1}_{U}\left(V(t+s, z, \omega)+f_{1}(t, z)+\lambda_{r}\left(f_{2}\right)(t, z)\right) d t \leq \varepsilon
$$

simultaneously for all $z \in[-N, N], r \in \mathbb{N}, s \in[0, T]$ and all $f_{1}, f_{2} \in \mathcal{C}_{(0, T]}^{h}(1$, $\mu, M)$.

The proof of the lemma is given in Section 7.

LEMMA 4.10. Let $b \in \mathbf{B}$. Then there exists a set $\Omega_{C} \subset \Omega$ with the following properties:

1) $\mathrm{P}\left(\Omega_{C}\right)=1$.

2) Let $\omega \in \Omega_{C}$. Then for any $T>0, h>1 / 2, M>0, \mu>0,0 \leq t_{1} \leq t_{2} \leq T$, $z \in \mathbb{R}, \theta \in \mathbf{B}$, function $\psi \in \mathcal{C}_{(0, T]}^{h}(1, \mu, M)$, any sequence $\left(s_{n}\right)_{n \in \mathbb{Z}_{+}}, s_{n} \in[0, T]$ 
converging to $s$ and any sequence of functions $f_{n} \in \mathcal{C}_{(0, T]}(h, 1, \mu, M)$ converging pointwise on $(0, T] \times \mathbb{R}$ to a limit $f$ we have

$$
\begin{gathered}
\lim _{n \rightarrow \infty} \int_{t_{1}}^{t_{2}} \theta(t) b\left(V\left(t+s_{n}, z, \omega\right)+f_{n}(t, z)+\lambda_{n}(\psi)(t, z)\right) d t \\
=\int_{t_{1}}^{t_{2}} \theta(t) b(V(t+s, z, \omega)+f(t, z)+\psi(t, z)) d t
\end{gathered}
$$

PROOF. The proof is based on the ideas from the proofs of [5], Lemmas 3.3, 3.4. Fix $h>1 / 2, \mu>0$ and integers $N, M, T>0$. Take arbitrary $\varepsilon>0$. Then there exists $\delta>0$ such that statement of Lemma 4.9 is satisfied.

By Lusin's theorem, there exists a continuous bounded function $\widetilde{b}: \mathbb{R} \rightarrow \mathbb{R}$ and an open set $U$ such that $|U|<\delta,\|\tilde{b}\|_{\infty} \leq 2\|b\|_{\infty}$ and $b(x)=\tilde{b}(x)$ for all $x \notin U$. Thus we have the bound

$$
|b(x)-\widetilde{b}(x)|=\mathbb{1}(x \in U)|b(x)-\widetilde{b}(x)| \leq 3\|b\|_{\infty} \mathbb{1}(x \in U) .
$$

Further, by Lemma 4.9, there exists a set $\Omega_{\varepsilon}$ with $\mathrm{P}\left(\Omega_{\varepsilon}\right) \geq 1-\varepsilon$ such that bound (4.21) holds on $\Omega_{\varepsilon}$. Take now any $\omega \in \Omega_{\varepsilon}, 0 \leq t_{1} \leq t_{2} \leq T, s, s_{n} \in[0, T], s_{n} \rightarrow s$, $z \in[-N, N], \theta \in \mathbf{B}$, a function $\psi \in \mathcal{C}_{(0, T]}^{h}(1, \mu, M)$ and any sequence of functions $f_{n} \in \mathcal{C}_{(0, T]}^{h}(1, \mu, M)$ converging pointwise to a limit $f \in \mathcal{C}_{(0, T]}^{h}(1, \mu, M)$. Taking into account (4.22), we have

$$
\begin{aligned}
\limsup _{n \rightarrow \infty} & \int_{t_{1}}^{t_{2}} \theta(t) b\left(V\left(t+s_{n}, z, \omega\right)+f_{n}(t, z)+\lambda_{n}(\psi)(t, z)\right) d t \\
\leq & \int_{t_{1}}^{t_{2}} \theta(t) \tilde{b}(V(t+s, z, \omega)+f(t, z)+\psi(t, z)) d t \\
& +3\|b\|_{\infty}\|\theta\|_{\infty} \limsup _{n \rightarrow \infty} \int_{t_{1}}^{t_{2}} \mathbb{1}_{U}\left(V\left(t+s_{n}, z, \omega\right)+f_{n}(t, z)+\lambda_{n}(\psi)(t, z)\right) d t \\
\leq & \int_{t_{1}}^{t_{2}} \theta(t) \widetilde{b}(V(t+s, z, \omega)+f(t, z)+\psi(t, z)) d t+3\|b\|_{\infty}\|\theta\|_{\infty} \varepsilon \\
\leq & \int_{t_{1}}^{t_{2}} \theta(t) b(V(t+s, z, \omega)+f(t, z)+\psi(t, z)) d t+3\|b\|_{\infty}\|\theta\|_{\infty} \varepsilon \\
& +3\|b\|_{\infty}\|\theta\|_{\infty} \int_{t_{1}}^{t_{2}} \mathbb{1}_{U}(V(t+s, z, \omega)+f(t, z)+\psi(t, z)) d t \\
\leq & \int_{t_{1}}^{t_{2}} \theta(t) b(V(t+s, z, \omega)+f(t, z)+\psi(t, z)) d t+6\|b\|_{\infty}\|\theta\|_{\infty} \varepsilon,
\end{aligned}
$$

where the second and the last inequalities follow from Lemma 4.9. 
By a similar argument, we have on $\Omega_{\varepsilon}$

$$
\begin{aligned}
\liminf _{n \rightarrow \infty} \int_{t_{1}}^{t_{2}} \theta(t) b\left(V\left(t+s_{n}, z, \omega\right)+f_{n}(t, z)+\lambda_{n}(\psi)(t, z)\right) d t \\
\quad \geq \int_{t_{1}}^{t_{2}} \theta(t) b(V(t+s, z, \omega)+f(t, z)+\psi(t, z)) d t-6\|b\|_{\infty}\|\theta\|_{\infty} \varepsilon .
\end{aligned}
$$

Since $\varepsilon$ was arbitrary, and since $\mathrm{P}\left(\Omega_{\varepsilon}\right) \geq 1-\varepsilon$ we see that there exists a set $\Omega(N, M, T, h, \mu)$ such that $\mathrm{P}(\Omega(N, M, T, h, \mu))=1$ and

$$
\begin{gathered}
\lim _{n \rightarrow \infty} \int_{t_{1}}^{t_{2}} \theta(t) b\left(V\left(t+s_{n}, z, \omega\right)+f_{n}(t, z)+\lambda_{n}(\psi)(t, z)\right) d t \\
=\int_{t_{1}}^{t_{2}} \theta(t) b(V(t+s, z, \omega)+f(t, z)+\psi(t, z)) d t
\end{gathered}
$$

for any $\omega \in \Omega(N, M, T, h, \mu), 0 \leq t_{1} \leq t_{2} \leq T, s, s_{n} \in[0, T], s_{n} \rightarrow s, \theta \in \mathbf{B}$, function $\psi \in \mathcal{C}_{(0, T]}^{h}(1, \mu, M), z \in[-N, N]$ and any sequence of functions $f_{n} \in$ $\mathcal{C}_{(0, T]}^{h}(1, \mu, M)$ converging pointwise to a limit $f \in \mathcal{C}_{(0, T]}^{h}(1, \mu, M)$.

To complete the proof of the lemma, it remains to take

$$
\Omega_{C}:=\bigcap \Omega(N, M, T, h, \mu),
$$

where the intersection is over all positive integers $N, M, T$ and rational $h>1 / 2$, $\mu>0$.

5. Proofs of Theorem 2.1 and Theorem 2.2(b). Most of the section is devoted to the proof of Theorem 2.1. Fix a bounded measurable function $b$. Without loss of generality and to ease the notation, we assume in this section that $\|b\|_{\infty} \leq 1$. Now with such $b$ at hand we take for the rest of the section

$$
\Omega^{\prime}:=\Omega_{E} \cap \Omega^{\prime \prime} \cap \Omega_{V} \cap \Omega_{C} \subset \Omega,
$$

where the set $\Omega_{E}$ is defined in the sketch of the proof of Theorem 2.1 in Section $2, \Omega^{\prime \prime}$ is from Theorem 2.3, $\Omega_{V}$ is from regularity Lemma 4.7 and $\Omega_{C}$ is from Lemma 4.10. Thus, on $\Omega^{\prime}$ the statements of the aforementioned theorems and lemmas are satisfied and $P\left(\Omega^{\prime}\right)=1$.

We begin this section with an easy observation.

Proposition 5.1. Let $s \geq 0, q \in \mathbf{B}(0+)$. Let $u_{s, q}$ be any solution to (2.2) that starts with initial condition $q$ at time $s$. Then $u_{s, q}(t, \cdot, \omega) \in \mathbf{B}(0+)$ for any $\omega \in \Omega^{\prime}, t \geq s$.

PROOF. This statement immediately follows from equation (2.2) and estimate (4.17). 
5.1. Existence part of Theorem 2.1. In this subsection, we present the proof of the existence part of Theorem 2.1. Our main tool is the following lemma that establishes continuity of solution to (2.2) with respect to the initial condition. Recall that the set $\Omega^{\prime}$ is defined in (5.1).

LEMMA 5.2. Let $\omega \in \Omega^{\prime}$. Let $\left(s_{n}\right)_{n \in \mathbb{Z}_{+}}, s_{n} \geq 0$ be a sequence that converges to $s$, as $n \rightarrow \infty$. Let $\left(q_{n}\right)_{n \in \mathbb{Z}_{+}}$be a sequence of measurable functions $\mathbb{R} \rightarrow \mathbb{R}$ such that $q_{n}(z) \rightarrow q(z)$ as $n \rightarrow \infty$ Lebesgue-almost everywhere in $z$. Assume that there exist $C>0, \mu>0$ such that for any $n \in \mathbb{Z}_{+}$one has

$$
\left|q_{n}(z)\right| \leq C\left(|z|^{\mu} \vee 1\right), \quad z \in \mathbb{R} .
$$

For each $n \in \mathbb{Z}_{+}$let $u_{s_{n}, q_{n}}(\cdot, \cdot, \omega)$ be a solution to $(2.2)$ that starts with the initial condition $q_{n}$ at time $s_{n}$.

Then there exists a solution $u_{s, q}(\cdot, \cdot, \omega)$ to (2.2) that starts with the initial condition $q$ at time $s$. Moreover, there exists a subsequence $\left(n_{k}\right)_{k \in \mathbb{Z}_{+}}$such that for any $t>0, z \in \mathbb{R}$ we have

$$
u_{s_{n_{k}}, q_{n_{k}}}\left(s_{n_{k}}+t, z, \omega\right) \rightarrow u_{s, q}(s+t, z, \omega) \quad \text { as } k \rightarrow \infty .
$$

This lemma implies that for any $\omega \in \Omega^{\prime}$ a sequence of solutions to equation (2.2) that start at time $s_{n}$ from the initial condition $q_{n}$ has a subsequence that converges pointwise to a solution of equation (2.2) that starts at time $s$ from the initial condition $q$.

Proof. Fix $\omega \in \Omega^{\prime}$, the sequences $\left(s_{n}\right)_{n \in \mathbb{Z}_{+}},\left(q_{n}\right)_{n \in \mathbb{Z}_{+}}$as in the lemma and also any $T>0$. By the definition of $u_{s_{n}, q_{n}}^{*}$ (recall equation (4.2)), we have for any $t \in(0, T], z \in \mathbb{R}$,

$$
\begin{aligned}
u_{s_{n}, q_{n}}^{*}(t, z)= & \int_{\mathbb{R}} p_{t}\left(z-z^{\prime}\right) q_{n}\left(z^{\prime}\right) d z^{\prime}+\int_{0}^{t} \int_{\mathbb{R}} p_{t-t^{\prime}}\left(z-z^{\prime}\right) b\left(u_{s_{n}, q_{n}}^{*}\left(t^{\prime}, z^{\prime}\right)\right) d z^{\prime} d t^{\prime} \\
& +V\left(s_{n}, t+s_{n}, z\right)
\end{aligned}
$$

and $u_{s_{n}, q_{n}}^{*}(0, z)=q_{n}(z)$. For $n \in \mathbb{Z}_{+}$, set

$$
h_{n}(t, z):=\int_{0}^{t} \int_{\mathbb{R}} p_{t-t^{\prime}}\left(z-z^{\prime}\right) b\left(u_{s_{n}, q_{n}}^{*}\left(t^{\prime}, z^{\prime}\right)\right) d z^{\prime} d t^{\prime}, \quad t \in[0, T], z \in \mathbb{R} .
$$

Clearly, the sequence $\left(h_{n}\right)_{n \in \mathbb{Z}_{+}}$is uniformly bounded. Indeed, for any $n \in \mathbb{Z}_{+}$we have $\left\|h_{n}\right\|_{\infty} \leq T\|b\|_{\infty}$. Further, it follows from Lemma 4.5 that for any $t_{1}, t_{2} \in$ $[0, T], z_{1}, z_{2} \in \mathbb{R}$,

$$
\left|h_{n}\left(t_{1}, z_{1}\right)-h_{n}\left(t_{2}, z_{2}\right)\right| \leq C\|b\|_{\infty}\left(\left|z_{2}-z_{1}\right|+\left|t_{2}-t_{1}\right|^{3 / 4}\right)
$$

for some $C=C(T)>0$ that is independent of $n$. Hence the Arzelà-Ascoli theorem for locally compact metric spaces (see, e.g., [10], Theorem 4.44) implies that there exists a subsequence $\left(n_{k}\right)_{k \in \mathbb{Z}_{+}}$, such that $h_{n_{k}}$ converges pointwise to some 
function $h$. We simplify the notation by assuming that we have already started with such a subsequence and that $n_{k}=k$. Hence, (5.2) yields for any $t_{1}, t_{2} \in[0, T]$ and $z_{1}, z_{2} \in \mathbb{R}$

$$
\left|h\left(t_{1}, z_{1}\right)-h\left(t_{2}, z_{2}\right)\right| \leq C\|b\|_{\infty}\left(\left|z_{2}-z_{1}\right|+\left|t_{2}-t_{1}\right|^{3 / 4}\right) .
$$

For $t \in(0, T], z \in \mathbb{R}$ put

$$
\begin{aligned}
& u_{s, q}^{*}(t, z):=\int_{\mathbb{R}} p_{t}\left(z-z^{\prime}\right) q\left(z^{\prime}\right) d z^{\prime}+h(t, z)+V(s, t+s, z), \\
& u_{s, q}^{*}(0, z):=q(z) .
\end{aligned}
$$

We claim now that $u_{s, q}^{*}$ is a solution to $(4.3)$ on $[0, T]$. Indeed, we observe that $u_{s_{n}, q_{n}}^{*}$ can be written as follows:

$$
u_{s_{n}, q_{n}}^{*}(t, z)=V\left(t+s_{n}, z\right)+g_{n}(t, z), \quad t \in[0, T], z \in \mathbb{R},
$$

where for $t \in[0, T], z \in \mathbb{R}$ we defined

$$
g_{n}(t, z):=\int_{\mathbb{R}} p_{t}\left(z-z^{\prime}\right) q_{n}\left(z^{\prime}\right) d z^{\prime}+h_{n}(t, z)-\mathcal{V}\left(0, s_{n}, t+s_{n}, z\right) .
$$

It follows from Lemma 4.6, Lemma 4.7 and inequality (5.2) that there exists $M>0$ such that for any $n \in \mathbb{Z}_{+}$the function $g_{n} \in \mathcal{C}_{(0, T]}^{3 / 4}(1, \mu, M)$. By our assumptions and the dominated convergence theorem, the first term at the right-hand side of (5.4) converges pointwise to $\int_{\mathbb{R}} p_{t}\left(z-z^{\prime}\right) q\left(z^{\prime}\right) d z^{\prime}$ for $(t, z) \in(0, T] \times \mathbb{R}$. By Lemma 4.7, $\mathcal{V}\left(0, s_{n}, t+s_{n}, z\right) \rightarrow \mathcal{V}(0, s, t+s, z)$ as $n \rightarrow \infty$ for $(t, z) \in[0, T] \times \mathbb{R}$. This together with $h_{n}$ converging pointwise to $h$ implies that

$$
\begin{aligned}
\lim _{n \rightarrow \infty} g_{n}(t, z) & =\int_{\mathbb{R}} p_{t}\left(z-z^{\prime}\right) q\left(z^{\prime}\right) d z^{\prime}+h(t, z)-\mathcal{V}(0, s, t+s, z) \\
& =: g(t, z), \quad t \in(0, T], z \in \mathbb{R} .
\end{aligned}
$$

On the other hand,

$$
\begin{aligned}
h(t, z) & =\lim _{n \rightarrow \infty} h_{n}(t, z) \\
& =\lim _{n \rightarrow \infty} \int_{\mathbb{R}} \int_{0}^{t} p_{t-t^{\prime}}\left(z-z^{\prime}\right) b\left(u_{s_{n}, q_{n}}^{*}\left(t^{\prime}, z^{\prime}\right)\right) d t^{\prime} d z^{\prime} \\
& =\lim _{n \rightarrow \infty} \int_{\mathbb{R}} \int_{0}^{t} p_{t-t^{\prime}}\left(z-z^{\prime}\right) b\left(V\left(t+s_{n}, z\right)+g_{n}(t, z)\right) d t^{\prime} d z^{\prime} .
\end{aligned}
$$

Note that the function $b$ is not necessarily continuous so we cannot pass to the limit directly. Therefore, to pass to the limit we employ Lemma 4.10 with the following set of parameters: $f_{n} \leftarrow g_{n}, f \leftarrow g, \psi \leftarrow 0, \theta \leftarrow p_{t-.}\left(z-z^{\prime}\right), t_{2} \leftarrow t$, $t_{1} \leftarrow 0$. Since $g_{n} \in \mathbf{H}_{T}(3 / 4,1, \mu, M)$ and since for fixed $t, z \neq z^{\prime}$ the function $t^{\prime} \mapsto$ $p_{t-t^{\prime}}\left(z-z^{\prime}\right)$ is bounded, we see that all conditions of Lemma 4.10 are satisfied. 
We apply the dominated convergence theorem (this is possible due to the fact that $b$ is bounded) and continue the identity above as follows:

$$
\begin{aligned}
h(t, z) & =\int_{\mathbb{R}} \int_{0}^{t} p_{t-t^{\prime}}\left(z-z^{\prime}\right) b(V(t+s, z)+g(t, z)) d t^{\prime} d z^{\prime} \\
& =\int_{\mathbb{R}} \int_{0}^{t} p_{t-t^{\prime}}\left(z-z^{\prime}\right) b\left(u_{s, q}^{*}\left(t^{\prime}, z^{\prime}\right)\right) d t^{\prime} d z^{\prime},
\end{aligned}
$$

where we also used that by $(5.3) u_{s, q}^{*}(t, z)=V(t+s, z)+g(t, z)$. Obtained identity (5.5), combined with (5.3), implies that $u_{s, q}^{*}$ is indeed a solution to (4.3). Hence the function $u_{s, q}(t, \cdot):=u_{s, q}^{*}(t+s, \cdot)$ solves equation (2.2) that starts with the initial condition $q$ at time $s$.

We note that the convergence of $g_{n}$ to $g$ and continuity of $V$ imply that

$$
\lim _{n \rightarrow \infty} u_{s_{n}, q_{n}}\left(s_{n}+t, z\right)=\lim _{n \rightarrow \infty} u_{s_{n}, q_{n}}^{*}(t, z)=u_{s, q}^{*}(t, z)=u_{s, q}(s+t, z)
$$

for any $t \in(0, T], z \in \mathbb{R}$. Finally, by the standard diagonalization argument, we see that there exists a subsequence $\left(n_{k}\right)$ such that identity (5.6) is valid for any $t \in(0, \infty)$.

PROOF OF EXISTENCE PART OF THEOREM 2.1. We recall that we have already fixed $\Xi$, a countable dense subset of $\mathbf{C}_{0}(\mathbb{R})$, and $\Theta$, a countable dense subset of $\mathbb{R}_{+}$(see the sketch of the proof of Theorem 2.1 in Section 2). Since $\Omega^{\prime} \subset \Omega_{E}$, we see that for any $\omega \in \Omega^{\prime}, s \in \Theta, q \in \Xi$ equation (2.2) has a solution that starts with the initial condition $q$ at time $s$.

Fix any $\omega \in \Omega^{\prime}$. Let $q$ now be an arbitrary element of $\mathbf{B}(0+)$, let $s \in \mathbb{R}$. Let $\left(q_{n}\right)_{n \in \mathbb{Z}_{+}}$be a sequence of elements in $\Xi$ that converge Lebesgue-almost everywhere to $q$ and such that for some $C>0, \mu>0$ one has $q_{n}(z) \leq C(|z| \vee 1)^{\mu}$ uniformly over all $n$. The existence of such a sequence is clear and can be shown by the standard argument. Let $\left(s_{n}\right)_{n \in \mathbb{Z}_{+}}$be a sequence of elements in $\Theta$ that converges to $s$. By above, equation (2.2) has a solution that starts with the initial condition $q_{n}$ at time $s_{n}$. Hence, by Lemma 5.2, equation (2.2) has a solution that starts with the initial condition $q$ at time $s$.

Since $q$ and $s$ were arbitrary elements of $\mathbf{B}(0+)$ and $\mathbb{R}_{+}$, respectively, this concludes the proof of the existence part of Theorem 2.1.

5.2. Uniqueness part of Theorem 2.1. Recall that by Remark 4.1 it is sufficient to show that on $\Omega^{\prime}$ equation (4.3) has a unique solution. This will straightforwardly imply that the original equation (2.2) has also a unique solution on $\Omega^{\prime}$.

Until the end of this section, we fix arbitrary $\omega \in \Omega^{\prime}, s \geq 0, q \in \mathbf{B}(0+)$. Without loss of generality, we assume $s \in[0,1]$. Let $v$ and $w$ be any two solutions to (4.3) with the initial condition $q$ for our fixed $\omega, s$. To prove the theorem, it is sufficient to show that $v(t, z)=w(t, z)$ for $z \in \mathbb{R}, t \in[0, T]$ for any $T>0$. We will verify this statement for $T=1$; the proof for other values of $T$ is exactly the same. 
We observe that for $t \in[0,1], z \in \mathbb{R}$ we have

$$
v(t, z)-w(t, z)=\int_{0}^{t} \int_{\mathbb{R}} p_{t-t^{\prime}}\left(z-z^{\prime}\right)\left(b\left(v\left(t^{\prime}, z^{\prime}\right)\right)-b\left(w\left(t^{\prime}, z^{\prime}\right)\right)\right) d z^{\prime} d t^{\prime} .
$$

We denote $\psi(t, z):=v(t, z)-w(t, z)$ and rewrite the above equation in the following form:

$$
\psi(t, z)=\int_{0}^{t} \int_{\mathbb{R}} p_{t-t^{\prime}}\left(z-z^{\prime}\right)\left(b\left(w\left(t^{\prime}, z^{\prime}\right)+\psi\left(t^{\prime}, z\right)\right)-b\left(w\left(t^{\prime}, z^{\prime}\right)\right)\right) d z^{\prime} d t^{\prime},
$$

where $t \in[0,1], z \in \mathbb{R}$. It is easy to check, that for any $r \in[0,1]$ the function $\psi$ satisfies also a more general equation

$$
\begin{aligned}
\psi(t, z)= & \int_{\mathbb{R}} p_{t-r}\left(z-z^{\prime}\right) \psi\left(r, z^{\prime}\right) d z^{\prime} \\
& +\int_{\mathbb{R}} \int_{r}^{t} p_{t-t^{\prime}}\left(z-z^{\prime}\right)\left(b\left(w\left(t^{\prime}, z^{\prime}\right)+\psi\left(t^{\prime}, z^{\prime}\right)\right)-b\left(w\left(t^{\prime}, z^{\prime}\right)\right)\right) d t^{\prime} d z^{\prime}, \\
\psi(0, z)= & \phi(z)
\end{aligned}
$$

where $t \in[r, 1], z \in \mathbb{R}$.

Our goal is to prove that the only solution to this equation with the initial condition $\phi(z)=0$ is identically zero (this would immediately imply uniqueness of solution to (4.3)). To show this, we have to analyze equation (5.7) with a more general class of initial conditions. Namely, we assume that the function $\phi \in \mathcal{C}_{\text {Lip }}^{b}$ (recall that the class $\mathcal{C}_{\text {Lip }}^{b}$ is introduced in Definition 4.1). Note also that the functions $\psi, v, w$ depend also on fixed $\omega, s, q$. In order not to overcrowd the notation, we write $\psi(t, z)$ for $\psi(t, z, \omega, s, q)$ and so on.

To show that equation (5.7) has only a trivial solution, we will need to control the norm of $\psi(t, \cdot)$. We will work with a weighted Hölder norm. The use of a weighted norm is natural here since we work with functions defined on a noncompact space $\mathbb{R}$. Thus, for a function $f: \mathbb{R} \rightarrow \mathbb{R}$ we put

$$
\|f\|_{0, w}=\sup _{z \in \mathbb{R}}|f(z)| e^{-|z|} .
$$

For $\delta>0$, consider a weighted Lipschitz coefficient of $f$,

$$
[f]_{1, \delta}:=\sup _{z_{1} \neq z_{2}} \frac{\left|f\left(z_{1}\right)-f\left(z_{2}\right)\right|}{\left|z_{1}-z_{2}\right| \Lambda_{\delta}\left(\left|z_{1}\right| \vee\left|z_{2}\right| \vee 1\right)} .
$$

Recall that the function $\Lambda_{\delta}$ was defined in (4.5).

Finally, define a weighted Lipschitz norm of $f$ by

$$
\|f\|_{1, \delta}:=\|f\|_{0, w}+[f]_{1, \delta} .
$$

We have to use the additional factor $z^{\delta}$ in the weight of the Lipschitz coefficient because this factor appears in the right-hand side of our main bound (2.4) in Theorem 2.3 (see also Lemma 5.6 below). 
The function $w$ can be represented as $w(t, z)=V(t+s, z)+g(t, z)$, where

$$
\begin{aligned}
g(t, z):= & \int_{\mathbb{R}} p_{t}\left(z-z^{\prime}\right) q\left(z^{\prime}\right) d z^{\prime}+\int_{0}^{t} \int_{\mathbb{R}} p_{t-t^{\prime}}\left(z-z^{\prime}\right) b\left(w\left(t^{\prime}, z^{\prime}\right)\right) d z^{\prime} d t^{\prime} \\
& -\mathcal{V}(0, s, t+s, z), \quad t \in[0,1], z \in \mathbb{R},
\end{aligned}
$$

and $\mathcal{V}$ was defined in (4.6). We used here the identity

$$
V(s, t+s, z)=\mathcal{V}(s, t+s, t+s, z)=\mathcal{V}(0, t+s, t+s, z)-\mathcal{V}(0, s, t+s, z) .
$$

The next two lemmas establish useful properties of the functions $g$ and $\psi$.

Lemma 5.3. The function $g$ defined in (5.8) belongs to $\mathcal{C}^{1-}(1,0+)$.

PROOF. The lemma follows immediately from Lemmas 4.5, 4.6, 4.7.

REMARK 5.4. Note that at any time $t>s$ a solution to $(2.2), u_{s, q}(t, \cdot)$, is much more regular than its initial condition $q \in \mathbf{B}(0+)$. Namely, $u_{s, q}(t, \cdot)$ is a Hölder function with exponent $1 / 2-$. If one starts with such a "regular" initial condition $q$ (Hölder with exponent $1 / 2-$ ), then it is possible to show that $g$ is more regular than it is shown in Lemma 5.3. Namely, $g \in \mathcal{C}^{1-}(3 / 4,0+)$. However, we will not use this improvement of regularity of $g$ in our proof and will continue to consider solutions to (2.2) that start from the initial condition $q \in \mathbf{B}(0+)$.

We will need to obtain the bounds on the norm of $\psi$ both in the weighted and in the standard Hölder spaces. As one might expect, there is a certain trade-off between having a singular weight and a better regularity.

Lemma 5.5. Assume that $\phi \in \mathcal{C}_{\text {Lip. }}^{b}$. Then any solution $\psi$ to (5.7) belongs to $\mathcal{C}^{1-}(1,0)$. Further, there exists a constant $C=C(\phi)$ such that

$$
\begin{aligned}
& \sup _{\substack{t \in[0,1] \\
z \in \mathbb{R}}}|\psi(t, z)| \leq C ; \\
& \sup _{\substack{t_{1}, t_{2} \in[0,1] \\
z_{1}, z_{2} \in \mathbb{R}}} \frac{\left|\psi\left(t_{1}, z_{1}\right)-\psi\left(t_{2}, z_{2}\right)\right|}{\left|t_{1}-t_{2}\right|^{1 / 2}+\left|z_{1}-z_{2}\right|} \leq C .
\end{aligned}
$$

That is, $\psi$ is a bounded function on $[0,1] \times \mathbb{R}$, which is Lipschitz in space and Hölder in time with exponent $1 / 2$. In particular, $\psi(t, \cdot) \in \mathcal{C}_{\mathrm{Lip}}^{b}$ for any $t \in[0,1]$.

Proof. Take in (5.7) $r=0$. Then, by Lemmas 4.5 and 4.6, $\psi \in \mathcal{C}^{1-}(1,0)$. Bound (5.9) obviously follows from boundedness of the functions $b$ and $\phi$. Estimate (5.10) is obtained by a straightforward application of Lemmas 4.5 and 4.6. 
The next lemma establishes "smoothing" properties of the operator

$$
(x(\cdot), s, t, z) \mapsto \int_{\mathbb{R}} \int_{0}^{s} p_{t-t^{\prime}}\left(z-z^{\prime}\right) b\left(V\left(t^{\prime}+s, z^{\prime}\right)+f\left(t^{\prime}, z^{\prime}\right)+x\left(z^{\prime}\right)\right) d t^{\prime} d z^{\prime},
$$

where $x \in \mathbf{B}$ and $0 \leq s \leq t$, simultaneously for all $f \in \mathcal{C}^{1-}(1,0+)$. To simplify the notation, further we will denote the sum of $V$ and the function $f:[0,1] \times \mathbb{R} \rightarrow \mathbb{R}$ (that will correspond to the drift term) by

$$
V_{f, s}(t, z):=V(t+s, z)+f(t, z), \quad t \in[0,1], z \in \mathbb{R} .
$$

Recall the definition of the difference of two Gaussian kernels $\Delta p_{t}$ from (4.4).

LEMMA 5.6. For any $\delta \in(0,1 / 6), N>0$, and any function $f \in \mathcal{C}^{1-}(1,0+)$ there exists a constant $C=C(\omega, N, f, \delta)<\infty$ such that for any $s \in[0,1], 0 \leq$ $t_{1} \leq t_{2} \leq t \leq 1, z, z_{1}, z_{2} \in \mathbb{R}$ and any $x, y \in \mathbf{B}$ with $\|x\|_{\infty},\|y\|_{\infty} \leq N$ we have

$$
\begin{aligned}
& \int_{\mathbb{R}}\left|\int_{t_{1}}^{t_{2}} p_{t-t^{\prime}}\left(z-z^{\prime}\right)\left(b\left(V_{f, s}\left(t^{\prime}, z^{\prime}\right)+x\left(z^{\prime}\right)\right)-b\left(V_{f, s}\left(t^{\prime}, z^{\prime}\right)+y\left(z^{\prime}\right)\right)\right) d t^{\prime}\right| d z^{\prime} \\
& \quad \leq C\|x-y\|_{0, w}\left|t_{2}-t_{1}\right|^{2 / 3-\delta} \Lambda_{\delta}(|z| \vee 1)
\end{aligned}
$$

$$
\begin{aligned}
& \int_{\mathbb{R}} \mid \int_{t_{1}}^{t_{2}} \Delta p_{t-t^{\prime}}\left(z_{1}-z^{\prime}, z_{2}-z^{\prime}\right) \\
& \quad \times\left(b\left(V_{f, s}\left(t^{\prime}, z^{\prime}\right)+x\left(z^{\prime}\right)\right)-b\left(V_{f, s}\left(t^{\prime}, z^{\prime}\right)+y\left(z^{\prime}\right)\right)\right) d t^{\prime} \mid d z^{\prime} \\
& \quad \leq C\|x-y\|_{0, w}\left(t-t_{1}\right)^{-1 / 2}\left|t_{2}-t_{1}\right|^{2 / 3-\delta}\left|z_{1}-z_{2}\right| \Lambda_{\delta}\left(\left|z_{1}\right| \vee\left|z_{2}\right| \vee 1\right)
\end{aligned}
$$

REMARK 5.7. If $f \in \mathcal{C}^{1-}(3 / 4,0+)$ (see Remark 5.4 where it is explained why this is relevant), then the operator (5.11) is more regular in time. Namely, the term $\left|t_{2}-t_{1}\right|$ in the right-hand side of (5.13) and (5.14) has the exponent $3 / 4-\delta$ instead of $2 / 3-\delta$.

Proof of Lemma 5.6. Fix $\delta \in(0,1 / 6), N>0$ and a function $f \in$ $\mathcal{C}^{1-}(1,0+)$. Consider a function

$$
B\left(r_{1}, r_{2}, s, \alpha, \beta, z\right):=\int_{r_{1}}^{r_{2}}\left(b\left(V_{f, s}\left(t^{\prime}, z\right)+\alpha\right)-b\left(V_{f, s}\left(t^{\prime}, z\right)+\beta\right)\right) d t^{\prime},
$$

defined for $\alpha, \beta, z \in \mathbb{R}, 0 \leq r_{1} \leq r_{2} \leq 1, s \in[0,1]$.

It follows from Theorem 2.3 that there exists a constant $C(\omega, N, f, \delta)$ such that for any $\alpha, \beta, z \in \mathbb{R},|\alpha|,|\beta| \leq N, 0 \leq r_{1} \leq r_{2} \leq 1, s \in[0,1]$ we have

$$
\left|B\left(r_{1}, r_{2}, s, \alpha, \beta, z\right)\right| \leq C(\omega, N, f, \delta)\left|r_{2}-r_{1}\right|^{2 / 3-\delta}|\alpha-\beta|\left(|z|^{\delta} \vee 1\right) .
$$

To simplify the notation, for the rest of the proof we drop the variables $\omega, N, f, \delta$ and write $C$ instead of $C(\omega, N, f, \delta)$. 
Fix $0 \leq t_{1} \leq t_{2} \leq 1$. Let $\left(t^{\prime}, z^{\prime}\right) \mapsto h\left(t^{\prime}, z^{\prime}\right), t^{\prime} \in\left[t_{1}, t_{2}\right], z^{\prime} \in \mathbb{R}$ be a continuously differentiable function in $t^{\prime}$ for $z^{\prime} \in \mathbb{R} \backslash E$, where the Lebesgue measure of $E$ is 0 . Then for any $\alpha, \beta \in \mathbb{R}, z^{\prime} \in \mathbb{R} \backslash E$ integration by parts gives

$$
\begin{array}{rl}
\int_{t_{1}}^{t_{2}} & h\left(t^{\prime}, z^{\prime}\right)\left(b\left(V_{f, s}\left(t^{\prime}, z^{\prime}\right)+\alpha\right)-b\left(V_{f, s}\left(t^{\prime}, z^{\prime}\right)+\beta\right)\right) d t^{\prime} \\
& =-\int_{t_{1}}^{t_{2}} h\left(t^{\prime}, z^{\prime}\right) d_{t^{\prime}} B\left(t^{\prime}, t_{2}, s, \alpha, \beta, z^{\prime}\right) \\
& =h\left(t_{1}, z^{\prime}\right) B\left(t_{1}, t_{2}, s, \alpha, \beta, z^{\prime}\right)+\int_{t_{1}}^{t_{2}} B\left(t^{\prime}, t_{2}, s, \alpha, \beta, z^{\prime}\right) \frac{\partial h}{\partial t^{\prime}}\left(t^{\prime}, z^{\prime}\right) d t^{\prime} .
\end{array}
$$

We integrate over $z^{\prime}$ and apply estimate (5.15) to derive for any $x, y \in \mathbf{B}$,

$$
\begin{aligned}
\int_{\mathbb{R}} \int_{t_{1}}^{t_{2}} h\left(t^{\prime}, z^{\prime}\right)\left(b\left(V_{f, s}\left(t^{\prime}, z^{\prime}\right)+x\left(z^{\prime}\right)\right)-b\left(V_{f, s}\left(t^{\prime}, z^{\prime}\right)+y\left(z^{\prime}\right)\right)\right) d t^{\prime} \\
\leq C\|x-y\|_{0, w}\left|t_{2}-t_{1}\right|^{2 / 3-\delta} \int_{\mathbb{R}}\left|h\left(t_{1}, z^{\prime}\right)\right| \Lambda_{\delta}\left(\left|z^{\prime}\right| \vee 1\right) d z^{\prime} \\
\quad+C\|x-y\|_{0, w} \int_{\mathbb{R}} \int_{t_{1}}^{t_{2}}\left|\frac{\partial h}{\partial t^{\prime}}\left(t^{\prime}, z^{\prime}\right)\right|\left|t_{2}-t^{\prime}\right|^{2 / 3-\delta} \Lambda_{\delta}\left(\left|z^{\prime}\right| \vee 1\right) d t^{\prime} d z^{\prime} .
\end{aligned}
$$

For any $t \geq t_{2}, z \in \mathbb{R}$, we can apply this formula to the function $h\left(t^{\prime}, z^{\prime}\right):=$ $p_{t-t^{\prime}}\left(z-z^{\prime}\right)$ (indeed, for $z^{\prime} \in \mathbb{R} \backslash z$ this function is continuously differentiable in $t^{\prime}$ ). Using estimates (4.10) and (4.11) from Lemma 4.4, we get (5.13). In a similar way, for $t \geq t_{2}, z_{1}, z_{2} \in \mathbb{R}$ we apply formula (5.16) to the function $h\left(t^{\prime}, z^{\prime}\right):=p_{t-t^{\prime}}\left(z_{1}-z^{\prime}\right)-p_{t-t^{\prime}}\left(z_{2}-z^{\prime}\right)$. Using estimates (4.12) and (4.13) from Lemma 4.4, we obtain (5.14).

REMARK 5.8. Let us explain how Lemmas 5.3, 5.5 and 5.6 will be used in the proof. Fix initial condition $\phi \in \mathcal{C}_{\text {Lip }}^{b}$. It follows from Lemma 5.5 that there exists a constant $C_{1}=C_{1}(\phi)$ such that inequalities (5.9) and (5.10) hold. Recall again that the solution $w$ can be represented as

$$
w(t, z)=V(t+s, z)+g(t, z),
$$

where $g$ was defined in (5.8). By Lemma 5.3, $g \in \mathcal{C}^{1-}(1,0+)$. Thus we can apply Lemma 5.6 with $f \leftarrow g$ and $N \leftarrow C_{1}$. We see that there exists a constant $C_{2}=$ $C_{2}\left(C_{1}, \phi\right)$ such that the estimates (5.13) and (5.14) are satisfied with $C_{2}$ instead of $C$. We will use further the constant $C_{\phi}:=\max \left(1, C_{1}, C_{2}\right)$.

Now, we apply Lemma 5.6 to analyze the behavior of $\psi$ on a small interval $\left[k 2^{-m},(k+1) 2^{-m}\right]$. More precisely, for any $t \in\left[k 2^{-m},(k+1) 2^{-m}\right]$ we will derive bounds on $\|\psi(t, \cdot)\|_{1, \delta}$ in terms of $\left\|\psi\left(k 2^{-m}, \cdot\right)\right\|_{1, \delta}$. This lemma will be crucial for the whole argument. Namely, we will just apply the bound from Lemma 5.9 consecutively $2^{m}$ times to prove later the uniqueness part of Theorem 2.1. 
LEMMA 5.9. For any $\delta \in(0,1 / 6)$ and any initial condition $\phi \in \mathcal{C}_{\text {Lip }}^{b}$, there exist constants $C=C(\delta, \phi), m_{0}=m_{0}(\delta, \phi)$ such that for any integers $m>m_{0}$, $r \in\left[0,2^{m}-1\right]$ we have the following estimate:

$$
\sup _{t \in\left[\frac{r}{2^{m}}, \frac{r+1}{2^{m}}\right]}\|\psi(t, \cdot)\|_{1, \delta} \leq C\left\|\psi\left(\frac{r}{2^{m}}, \cdot\right)\right\|_{1, \delta}+C e^{-2^{m /(2 \delta)}} .
$$

In particular,

$$
\left\|\psi\left(\frac{r+1}{2^{m}}, \cdot\right)\right\|_{1, \delta} \leq C\left\|\psi\left(\frac{r}{2^{m}}, \cdot\right)\right\|_{1, \delta}+C e^{-2^{m /(2 \delta)}} .
$$

Proof. Fix $\delta \in(0,1 / 6)$, the initial condition $\phi$ and integer $m>0$. All the constants that will appear in the proof will depend only on $\delta$ and $\phi$ but not on $m$ or $r$.

To simplify the notation, we will show (5.18) for $r=0$. The proof for other values of $r=1,2, \ldots, 2^{m}-1$ is exactly the same.

Recall that we already know from Lemma 5.5 and Remark 5.8 that

$$
\sup _{\substack{t_{1}, t_{2} \in\left[0,2^{-m}\right] \\ z \in \mathbb{R}}} \frac{\left|\psi\left(t_{1}, z\right)-\psi\left(t_{2}, z\right)\right|}{\left|t_{1}-t_{2}\right|^{1 / 2}} \leq C_{\phi} .
$$

This bound is rather rough; our goal is to obtain a much finer bound that we can later iterate over $r$. We will show in the proof that if $\|\psi(0, \cdot)\|_{1, \delta}$ is small, then the left-hand side of (5.20) is also very small. This would imply (5.18).

Our proof strategy consists of three steps. First, following a standard technique (see, e.g., [5], proof of Lemma 3.1), we show that it is sufficient to estimate the supremum in the left-hand side of (5.20) only for those $t_{1}, t_{2} \in\left[0,2^{-m}\right]$ that are dyadic neighbors. This would imply a corresponding bound for any $t_{1}, t_{2} \in\left[0,2^{-m}\right]$. As is common in the PDE literature, to get a "time" bound we need to obtain first a "space" bound. This is done in the second step using approximation technique and estimate (5.14). Finally, using again approximation technique and estimate (5.13) we get the required "time" bound (5.20) with a much smaller constant.

In the proof of the theorem, we will be working with binomial partitions of the interval $[0,1]$. So, for integers $n \geq 0, k \in\left[0,2^{n}\right]$ put

$$
t_{n}^{k}:=k 2^{-n} ; \quad \operatorname{Lip}_{n}^{k}:=\left[\psi\left(t_{n}^{k}, \cdot\right)\right]_{1, \delta} .
$$

By Lemma 5.5, $\operatorname{Lip}_{n}^{k}$ are finite for any $n \geq 0, k \in\left[0,2^{n}\right]$.

As explained above, we will consider the differences $\left|\psi\left(t_{1}, z\right)-\psi\left(t_{2}, z\right)\right|$, $t_{1}, t_{2} \in\left[0,2^{-m}\right]$, where $t_{1}$ and $t_{2}$ are dyadic neighbors. Thus we define $\alpha$ as the smallest number such that for any integers $n \geq m, k \in\left[0,2^{n-m}-1\right]$ we have

$$
\left\|\psi\left(\frac{k+1}{2^{n}}, \cdot\right)-\psi\left(\frac{k}{2^{n}}, \cdot\right)\right\|_{0, w} \leq \alpha 2^{-n / 2} .
$$


Note that such an $\alpha$ exists and is finite. Indeed thanks to Remark 5.8, the left-hand side of (5.22) is bounded by $C_{\phi} 2^{-n / 2}$.

Consider a binary notation of $k 2^{-n}$, where $k \in\left[0,2^{n-m}-1\right]$. We have $k 2^{-n}=$ $\sum_{i=m+1}^{n} d_{i} 2^{-i}$, where each $d_{i}$ equals 0 or 1 . Define approximations of $k 2^{-n}$ by

$$
k_{m}:=0, k_{j}:=\sum_{i=m+1}^{j} d_{i} 2^{-i}, \quad j \in[m+1, n] .
$$

It follows from the definition that either $k_{j}=k_{j-1}$ or $k_{j}=k_{j-1}+2^{-j}$. Therefore, we can apply estimate (5.22) $n-m$ times to derive

$$
\left\|\psi\left(\frac{k}{2^{n}}, \cdot\right)-\psi(0, \cdot)\right\|_{0, w} \leq \sum_{j=m+1}^{n}\left\|\psi\left(k_{j}, \cdot\right)-\psi\left(k_{j-1}, \cdot\right)\right\|_{0, w} \leq \alpha \sum_{j=m+1}^{n} 2^{-j / 2} .
$$

Hence, for any $n \geq m$ and $0 \leq k \leq 2^{n-m}-1$ we get

$$
\left\|\psi\left(\frac{k}{2^{n}}, \cdot\right)\right\|_{0, w} \leq\|\psi(0, \cdot)\|_{0, w}+3 \alpha 2^{-m / 2} .
$$

Since the function $\psi$ is continuous, we get the following bound for any $t \in$ $\left[0,2^{-m}\right]$ :

$$
\|\psi(t, \cdot)\|_{0, w} \leq\|\psi(0, \cdot)\|_{0, w}+3 \alpha 2^{-m / 2} .
$$

Thus we can effectively bound $\|\psi(t, \cdot)\|_{0, w}$ for any $t \in\left[0,2^{-m}\right]$.

To approximate the solutions to (5.7), we consider piecewise approximations defined above in (4.1). Namely, we introduce a sequence of piecewise-constant (in time) functions

$$
\psi_{n}(\cdot, z):=\lambda_{n}(\psi(\cdot, z)), \quad z \in \mathbb{R},
$$

where $n \geq m$. We see that $\psi_{n}(t, z)$ is equal to $\psi\left((k+1) 2^{-n}, z\right)$ whenever $t \in\left(k 2^{-n},(k+1) 2^{-n}\right], k=0,1, \ldots, 2^{n-m}-1$. In particular, the function $\psi_{m}(t, z)$ is constant in $t$ on the interval $\left(0,2^{-m}\right]$.

We start with an estimation of the weighted Lipschitz coefficient (with respect to the space variable) of the function $\psi$. We want to do it in all binary points of our initial interval $\left[0,2^{-m}\right]$, that is, in all points of the form $t_{n}^{k}=k 2^{-n}$. Here, $n \geq m$, $0 \leq k \leq 2^{n-m}-1$. Recall the definition of the function $g$ and the process $V_{g, s}$ given at (5.8) and (5.12). respectively. Taking into account (5.17), we derive from (5.7)

$$
\psi\left(t_{n}^{k+1}, z_{1}\right)-\psi\left(t_{n}^{k+1}, z_{2}\right)=I_{1}+I_{2},
$$

where

$$
I_{1}:=\int_{\mathbb{R}} p_{2^{-n}}\left(z^{\prime}\right)\left(\psi\left(t_{n}^{k}, z_{1}-z^{\prime}\right)-\psi\left(t_{n}^{k}, z_{2}-z^{\prime}\right)\right) d z^{\prime}
$$

and

$$
I_{2}:=\int_{\mathbb{R}} \int_{t_{n}^{k}}^{t_{n}^{k+1}} \Delta p_{t_{n}^{k+1}-t}\left(z_{1}-z^{\prime}, z_{2}-z^{\prime}\right)\left(b\left(V_{g, s}\left(t, z^{\prime}\right)+\psi\left(t, z^{\prime}\right)\right)-b\left(V_{g, s}\left(t, z^{\prime}\right)\right)\right) d t d z^{\prime} .
$$


First let us bound $I_{1}$. By definition of $\operatorname{Lip}_{n}^{k}$ [see (5.21)], we have

$$
\begin{aligned}
\left|I_{1}\right| & \leq \operatorname{Lip}_{n}^{k}\left|z_{1}-z_{2}\right| \int_{\mathbb{R}} p_{2^{-n}}\left(z^{\prime}\right) \Lambda_{\delta}\left(\left|z_{1}-z^{\prime}\right| \vee\left|z_{2}-z^{\prime}\right| \vee 1\right) d z^{\prime} \\
& \leq \operatorname{Lip}_{n}^{k}\left|z_{1}-z_{2}\right| \Lambda_{\delta}\left(\left|z_{1}\right| \vee\left|z_{2}\right| \vee 1\right) \int_{\mathbb{R}} p_{2^{-n}}\left(z^{\prime}\right) e^{\left|z^{\prime}\right|}\left(\left|z^{\prime}\right|^{\delta}+1\right) d z^{\prime} \\
& \leq \operatorname{Lip}_{n}^{k}\left|z_{1}-z_{2}\right| \Lambda_{\delta}\left(\left|z_{1}\right| \vee\left|z_{2}\right| \vee 1\right) e^{2^{-n / 2}}\left(1+C 2^{-n \delta / 2}\right),
\end{aligned}
$$

where in the second inequality we also used the fact that the function $\Lambda$ is increasing and $\left|z_{1}-z^{\prime}\right| \vee\left|z_{2}-z^{\prime}\right| \vee 1 \leq\left(\left|z_{1}\right| \vee\left|z_{2}\right| \vee 1\right)+\left|z^{\prime}\right|$.

To handle $I_{2}$, we apply Lemma 4.10 with the following set of parameters: $f_{n} \leftarrow$ $g, f \leftarrow g, \psi \leftarrow \psi, \theta \leftarrow \Delta p_{t_{n}^{k+1}-.}\left(z_{1}-z^{\prime}, z_{2}-z^{\prime}\right), s_{n} \leftarrow s, t_{2} \leftarrow t_{n}^{k+1}, t_{1} \leftarrow t_{n}^{k}$. Since $\psi, g \in \mathcal{C}^{1-}\left(1,0+\right.$ ) (see Lemmas 5.3 and 5.5), and since for fixed $z^{\prime} \neq z_{1}, z_{2}$ the function $\Delta p_{t_{n}^{k+1} \text {. }}\left(z_{1}-z^{\prime}, z_{2}-z^{\prime}\right)$ is bounded, we see that all the conditions of Lemma 4.10 are satisfied. Since the function $b$ is also bounded, we can apply dominated convergence theorem to obtain

$$
\begin{aligned}
I_{2}= & \lim _{l \rightarrow \infty} \int_{\mathbb{R}} \int_{t_{n}^{k}}^{t_{n}^{k+1}} \Delta p_{t_{n}^{k+1}-t}\left(z_{1}-z^{\prime}, z_{2}-z^{\prime}\right) \\
& \times\left(b\left(V_{g, s}\left(t, z^{\prime}\right)+\psi_{l}\left(t, z^{\prime}\right)\right)-b\left(V_{g, s}\left(t, z^{\prime}\right)\right)\right) d t d z^{\prime} \\
= & \int_{\mathbb{R}} \int_{t_{n}^{k}}^{t_{n}^{k+1}} \Delta p_{t_{n}^{k+1}-t}\left(z_{1}-z^{\prime}, z_{2}-z^{\prime}\right) \\
& \times\left(b\left(V_{g, s}\left(t, z^{\prime}\right)+\psi_{n}\left(t, z^{\prime}\right)\right)-b\left(V_{g, s}\left(t, z^{\prime}\right)\right)\right) d t d z^{\prime} \\
& +\sum_{l=n}^{\infty} I_{22}(l) \\
= & : I_{21}+\sum_{l=n}^{\infty} I_{22}(l),
\end{aligned}
$$

where we denoted

$$
\begin{aligned}
I_{22}(l):= & \int_{\mathbb{R}} \int_{t_{n}^{k}}^{t_{n}^{k+1}} \Delta p_{t_{n}^{k+1}-t}\left(z_{1}-z^{\prime}, z_{2}-z^{\prime}\right) \\
& \times\left(b\left(V_{g, s}\left(t, z^{\prime}\right)+\psi_{l+1}\left(t, z^{\prime}\right)\right)-b\left(V_{g, s}\left(t, z^{\prime}\right)+\psi_{l}\left(t, z^{\prime}\right)\right)\right) d t d z^{\prime} .
\end{aligned}
$$

Thus Lemma 4.10 allowed us to pass from continuous function $\psi$ to its piecewise-constant approximations $\psi_{l}$. This is crucial due to the fact that our main tool, Lemma 5.6, works only for constant in $t$ functions $x$ and $y$.

Estimation of $I_{21}$ is straightforward. It follows from the definition of approximation $\psi_{n}$, that $\psi_{n}(t, z)=\psi\left(t_{n}^{k+1}, z\right)$ for any $t \in\left(t_{n}^{k}, t_{n}^{k+1}\right], z \in \mathbb{R}$. Taking into 
account Remark 5.8, we make use of the bounds in (5.14) and (5.23) to obtain

$$
\begin{aligned}
\left|I_{21}\right| & \leq C_{\phi}\left|z_{1}-z_{2}\right| 2^{-(1 / 6-\delta) n}\left\|\psi\left(t_{n}^{k+1}, \cdot\right)\right\|_{0, w} \Lambda_{\delta}\left(\left|z_{1}\right| \vee\left|z_{2}\right| \vee 1\right) \\
& \leq C C_{\phi}\left|z_{1}-z_{2}\right| 2^{-(1 / 6-\delta) n} \Lambda_{\delta}\left(\left|z_{1}\right| \vee\left|z_{2}\right| \vee 1\right)\left(\|\psi(0, \cdot)\|_{0, w}+\alpha 2^{-m / 2}\right)
\end{aligned}
$$

Now let us do the most tricky part and work with term $I_{22}(l)$ in (5.24). As mentioned above, the functions $\psi_{l}$ and $\psi_{l+1}$ are piecewise-constant. Thus we split the integral over $\left(t_{n}^{k}, t_{n}^{k+1}\right]$ into $2^{l-n}$ integrals over smaller subintervals $\left(t_{l}^{i}, t_{l}^{i+1}\right]$, $k 2^{l-n} \leq i<(k+1) 2^{l-n}$. On each such subinterval function, $\psi_{l}$ is constant in $t$ and is equal to $\psi\left(t_{l}^{i+1}, z\right)$. The function $\psi_{l+1}$ also equal to the same value $\psi\left(t_{l}^{i+1}, z\right)$ for $t \in\left(t_{l}^{i+1 / 2}, t_{l}^{i+1}\right]$ and to $\psi\left(t_{l}^{i+1 / 2}, z\right)$ for $t \in\left(t_{l}^{i}, t_{l}^{i+1 / 2}\right]$. Thus we get

$$
\begin{aligned}
I_{22}(l)= & \sum_{i=k 2^{l-n}}^{(k+1) 2^{l-n}-1} \int_{\mathbb{R}} \int_{t_{l}^{i}}^{t_{l}^{i+1}} \Delta p_{t_{n}^{k+1}-t}\left(z_{1}-z^{\prime}, z_{2}-z^{\prime}\right) \\
& \times\left(b\left(V_{g, s}\left(t, z^{\prime}\right)+\psi_{l+1}\left(t, z^{\prime}\right)\right)-b\left(V_{g, s}\left(t, z^{\prime}\right)+\psi_{l}\left(t, z^{\prime}\right)\right)\right) d t d z^{\prime} \\
= & \sum_{i=k 2^{l-n}}^{(k+1) 2^{l-n}-1} \int_{\mathbb{R}} \int_{t_{l}^{i}}^{t_{l}^{i+1 / 2}} \Delta p_{t_{n}^{k+1}-t}\left(z_{1}-z^{\prime}, z_{2}-z^{\prime}\right) \\
& \times\left(b\left(V_{g, s}\left(t, z^{\prime}\right)+\psi\left(t_{l}^{i+1 / 2}, z^{\prime}\right)\right)-b\left(V_{g, s}\left(t, z^{\prime}\right)+\psi\left(t_{l}^{i+1}, z\right)\right)\right) d t d z^{\prime} .
\end{aligned}
$$

We apply our main estimate (5.14) and assumption (5.22) to this identity. We derive

$$
\begin{aligned}
\left|I_{22}(l)\right| \leq & C_{\phi}\left|z_{1}-z_{2}\right| \Lambda_{\delta}\left(\left|z_{1}\right| \vee\left|z_{2}\right| \vee 1\right) 2^{-l(2 / 3-\delta)} \\
& \times \sum_{i=k 2^{l-n}}^{(k+1) 2^{l-n}-1}\left(t_{n}^{k+1}-t_{l}^{i}\right)^{-1 / 2}\left\|\psi\left(t_{l}^{i+1}, \cdot\right)-\psi\left(t_{l}^{i+1 / 2}, \cdot\right)\right\|_{0, w} \\
\leq & C_{\phi}\left|z_{1}-z_{2}\right| \Lambda_{\delta}\left(\left|z_{1}\right| \vee\left|z_{2}\right| \vee 1\right) \alpha 2^{-l(2 / 3-\delta)} \sum_{i=k 2^{l-n}}^{(k+1) 2^{l-n}-1}\left((k+1) 2^{l-n}-i\right)^{-1 / 2} \\
\leq & C C_{\phi}\left|z_{1}-z_{2}\right| \Lambda_{\delta}\left(\left|z_{1}\right| \vee\left|z_{2}\right| \vee 1\right) \alpha 2^{-l(1 / 6-\delta)} 2^{-n / 2} .
\end{aligned}
$$

Therefore,

$$
\sum_{l=n}^{\infty} I_{22}(l) \leq C C_{\phi} \alpha\left|z_{1}-z_{2}\right| \Lambda_{\delta}\left(\left|z_{1}\right| \vee\left|z_{2}\right| \vee 1\right) 2^{-n(2 / 3-\delta)}
$$

Combining (5.24), (5.25), (5.26), (5.27) and (5.28), we finally obtain

$$
\begin{aligned}
\operatorname{Lip}_{n}^{k+1} \leq & e^{2^{-n / 2}}\left(1+C 2^{-n \delta / 2}\right) \operatorname{Lip}_{n}^{k}+C C_{\phi} 2^{-n(1 / 6-\delta)}\left(\|\psi(0, \cdot)\|_{0, w}+\alpha 2^{-m / 2}\right) \\
& +C C_{\phi} \alpha 2^{-n(2 / 3-\delta)} \\
= & : a_{n} \operatorname{Lip}_{n}^{k}+b_{n} .
\end{aligned}
$$


We consider again binary approximations to $t_{n}^{k}$ and employ again the same argument as we used in the proof of (5.23). We get

$$
\operatorname{Lip}_{n}^{k+1} \leq\left(\prod_{i=m+1}^{n} a_{i}\right)\left(\operatorname{Lip}_{m}^{0}+\sum_{i=m+1}^{n} b_{i}\right)
$$

Since

$$
\prod_{i=m+1}^{n} a_{i}=\prod_{i=m+1}^{n} e^{2^{-i / 2}}\left(1+C 2^{-i \delta / 2}\right) \leq \exp \left(\sum_{i=m+1}^{n}\left(2^{-i / 2}+C 2^{-i \delta / 2}\right)\right)
$$

is bounded uniformly in $n$, we derive

$$
\operatorname{Lip}_{n}^{k} \leq C C_{\phi}\|\psi(0, \cdot)\|_{1, \delta}+C C_{\phi} \alpha 2^{-m(2 / 3-\delta)}, \quad n \geq m, k \in\left[0,2^{n-m}-1\right] .
$$

Using again continuity of the function $\psi$ and the fact that the constants $C, C_{\phi}$ in the bound above do not depend on $k, n$, we arrive to the bound

$$
[\psi(t, \cdot)]_{1, \delta} \leq C C_{\phi}\|\psi(0, \cdot)\|_{1, \delta}+C C_{\phi} \alpha 2^{-m(2 / 3-\delta)}, \quad t \in\left[0,2^{-m}\right] .
$$

Now we return to the main line of the proof. Recall that our aim is to estimate left-hand side of (5.22) and bound $\alpha$. We treat large $|z|$ and small $|z|$ differently. Therefore, we fix a large threshold $M>1$. The precise value of $M$ will depend on $m$ and will be chosen later.

If $|z|$ is large enough, we use very rough estimates from Lemma 5.5:

$$
\sup _{|z| \geq M} e^{-|z|}\left|\left(\psi\left(t_{n}^{k+1}, z\right)-\psi\left(t_{n}^{k}, z\right)\right)\right| \leq C_{\phi} e^{-M} 2^{-n / 2} .
$$

For small $z$, we estimate the same quantity more precisely. Arguing similarly to (5.26) and using (5.7) and Lemma 4.10, we obtain for any $z \in \mathbb{R}$, and integers $n \geq m, k \in\left[0,2^{n-m}-1\right]$,

$$
\begin{aligned}
& \psi\left(t_{n}^{k+1}, z\right)-\psi\left(t_{n}^{k}, z\right) \\
&=\int_{\mathbb{R}} p_{2^{-n}}\left(z^{\prime}\right)\left(\psi\left(t_{n}^{k}, z-z^{\prime}\right)-\psi\left(t_{n}^{k}, z\right)\right) d z^{\prime} \\
& \quad+\int_{\mathbb{R}} \int_{t_{n}^{k}}^{t_{n}^{k+1}} p_{t_{n}^{k+1}-t}\left(z-z^{\prime}\right) \\
& \quad \times\left(b\left(V_{g, s}\left(t, z^{\prime}\right)+\psi_{n}\left(t, z^{\prime}\right)\right)-b\left(V_{g, s}\left(t, z^{\prime}\right)\right)\right) d t d z^{\prime} \\
& \quad+\sum_{l=n}^{\infty} \int_{\mathbb{R}} \int_{t_{n}^{k}}^{t_{n}^{k+1}} p_{t_{n}^{k+1}-t}\left(z-z^{\prime}\right) \\
& \quad \times\left(b\left(V_{g, s}(t, z)+\psi_{l+1}(t, z)\right)-b\left(V_{g, s}(t, z)+\psi_{l}(t, z)\right)\right) d t d z^{\prime} \\
&=: J_{1}(k, z)+J_{2}(k, z)+J_{3}(k, z) .
\end{aligned}
$$


By the definition of $\operatorname{Lip}_{n}^{k}$ and (5.29), we have

$$
\begin{aligned}
\sup _{|z| \leq M} e^{-|z|}\left|J_{1}(k, z)\right| & \leq C \operatorname{Lip}_{n}^{k} M^{\delta} 2^{-n / 2} \\
& \leq C C_{\phi} M^{\delta} 2^{-n / 2}\left(\|\psi(0, \cdot)\|_{1, \delta}+\alpha 2^{-m(2 / 3-\delta)}\right) .
\end{aligned}
$$

To bound $e^{-|z|} J_{2}(k, z)$, we apply estimate (5.13). We get

$$
\begin{aligned}
\sup _{|z| \leq M} e^{-|z|}\left|J_{2}(k, z)\right| & \\
= & \sup _{|z| \leq M} e^{-|z|} \mid \int_{\mathbb{R}} \int_{t_{n}^{k}}^{t_{n}^{k+1}} p_{t_{n}^{k+1}-t}\left(z-z^{\prime}\right) \\
& \times\left(b\left(V_{g, s}\left(t, z^{\prime}\right)+\psi\left(t_{n}^{k+1}, z^{\prime}\right)\right)-b\left(V_{g, s}\left(t, z^{\prime}\right)\right)\right) d t d z^{\prime} \mid \\
\leq & C_{\phi} M^{\delta}\left\|\psi\left(t_{n}^{k+1}, \cdot\right)\right\|_{0, w^{2}} 2^{-n(2 / 3-\delta)} .
\end{aligned}
$$

Hence, by (5.23) we have

$$
\sup _{|z| \leq M} e^{-|z|}\left|J_{2}(k, z)\right| \leq C C_{\phi} 2^{-n(2 / 3-\delta)} M^{\delta}\left(\|\psi(0, \cdot)\|_{0, w}+\alpha 2^{-m / 2}\right) .
$$

Finally, let us work with $J_{3}$. We estimate it using (5.13) and (5.22). We derive (similar to derivation of the bound on $I_{22}(l)$ )

$$
\begin{aligned}
& \sup _{|z| \leq M} e^{-|z|}\left|J_{3}(k, z)\right| \\
& \quad \leq C_{\phi} M^{\delta} \sum_{l=n}^{\infty} \sum_{i=k 2^{l-n}}^{(k+1) 2^{l-n}-1} 2^{-l(2 / 3-\delta)}\left\|\psi\left(t_{l}^{i+1}, \cdot\right)-\psi\left(t_{l}^{i+1 / 2}, \cdot\right)\right\|_{0, w} \\
& \leq C_{\phi} M^{\delta} \alpha \sum_{l=n}^{\infty} 2^{-n} 2^{-l(1 / 6-\delta)} \\
& \quad \leq C C_{\phi} M^{\delta} \alpha 2^{-n(7 / 6-\delta)} .
\end{aligned}
$$

Thus it remains just to combine (5.30) and the obtained estimates of terms in the right-hand side of (5.31) (we use (5.32), (5.33) and (5.34)) to obtain for any integers $n \geq m, k \in\left[0,2^{n-m}-1\right]$,

$$
\begin{aligned}
\left\|\psi\left(t_{n}^{k+1}, \cdot\right)-\psi\left(t_{n}^{k}, \cdot\right)\right\|_{0, w} \leq & C C_{\phi} 2^{-n / 2} M^{\delta}\left(\|\psi(0, \cdot)\|_{1, \delta}+\alpha 2^{-m(2 / 3-\delta)}\right) \\
& +C_{\phi} e^{-M} 2^{-n / 2}
\end{aligned}
$$

Comparing this with (5.22) and using the definition of $\alpha$, we get

$$
\alpha \leq C C_{\phi} M^{\delta}\left(\|\psi(0, \cdot)\|_{1, \delta}+\alpha 2^{-m(2 / 3-\delta)}\right)+C_{\phi} e^{-M} .
$$


Now we pick $M=2^{m /(2 \delta)}$ and rewrite the obtained bound

$$
\alpha \leq C C_{\phi} 2^{-m(1 / 6-\delta)} \alpha+C C_{\phi} 2^{m / 2}\|\psi(0, \cdot)\|_{1, \delta}+C_{\phi} e^{-2^{m /(2 \delta)}} .
$$

Recall that the constants $C, C_{\phi}$ do not depend on $m$. Thus, if we choose now $m_{0}=$ $m_{0}(\delta, \phi)$ large enough such that $C C_{\phi} 2^{-m_{0}(1 / 6-\delta)}<1 / 2$, then for any $m \geq m_{0}$ we finally deduce

$$
\alpha \leq C C_{\phi} 2^{m / 2}\|\psi(0, \cdot)\|_{1, \delta}+C C_{\phi} e^{-2^{m /(2 \delta)}}
$$

Let us stress once again, that the constants that appear in the right-hand side of the above equation $\left(C, C_{\phi}, m_{0}\right)$ depend only on $\delta$ and $\phi$, but not on $r$ ( $r$ was assumed to be equal to 0 in the beginning of the proof). The proof for other values of $r$ is exactly the same with exactly the same final constants $C, C_{\phi}, m_{0}$.

To complete the proof, it remains just to substitute the obtained estimate of $\alpha$ (5.35) into estimates (5.23) and (5.29). Thus we get the following final bound on the Hölder norm of $\psi(t, \cdot)$ :

$$
\|\psi(t, \cdot)\|_{1, \delta}=\|\psi(t, \cdot)\|_{0, w}+[\psi(t, \cdot)]_{1, \delta} \leq C C_{\phi}\|\psi(0, \cdot)\|_{1, \delta}+C C_{\phi} e^{-2^{m /(2 \delta)}}
$$

valid for all $t \in\left[0,2^{-m}\right]$. This proves (5.18). Estimate (5.19) is an immediate corollary of (5.18).

Now we can straightforwardly estimate the behavior of $\psi$ on bigger intervals and give a proof of uniqueness part of Theorem 2.1 .

PROOF OF UNIQUENESS PART OF THEOREM 2.1. We apply Lemma 5.9 with $\delta=1 / 10$ and zero initial condition $\phi=0$. For large enough $m \geq m_{0}$, we apply bound (5.19) consequently $2^{m}$ times. We get that there exists a constant $C>0$ such that for any integer $k \in\left[0,2^{m}\right]$,

$$
\left\|\psi\left(k 2^{-m}, \cdot\right)\right\|_{1, \delta} \leq C^{2^{m}} \exp \left(-2^{m /(2 \delta)}\right) .
$$

Note that the constant $C$ does not depend on $m$ or $k$. Therefore, by letting $m \rightarrow \infty$ we get $\|\psi(t, \cdot)\|_{1, \delta}=0$ for any dyadic $t \in[0,1]$. By the continuity of $\psi$, we see that $\|\psi(t, \cdot)\|_{1, \delta}=0$ for any $t \in[0,1]$, which implies that $\psi$ is identically 0 on $[0,1] \times \mathbb{R}$.

Thus equation (5.7) has only the trivial solution and, therefore, on $\Omega^{\prime}$ equation (2.2) has a unique solution. This solution is in $\mathbf{B}(0+)$ by Proposition 5.1.

Finally, let us prove the last part of the theorem. Let $q_{1}, q_{2} \in \mathbf{B}(0+)$ be two initial conditions and $q_{1}(z)=q_{2}(z)$ Lebesgue-almost everywhere. Let $u_{s, q_{1}}$ and $u_{s, q_{2}}$ be the solutions to (2.2) that start with initial conditions $q_{1}$ and $q_{2}$ correspondingly. Note that for $t \in(0,1], z \in \mathbb{R}$ we have

$$
\int_{\mathbb{R}} p_{t}\left(z-z^{\prime}\right) q_{1}\left(z^{\prime}\right) d z^{\prime}=\int_{\mathbb{R}} p_{t}\left(z-z^{\prime}\right) q_{2}\left(z^{\prime}\right) d z^{\prime}
$$


Consider now the function $v(t, z):=u_{s, q_{2}}(t, z, \omega)$ for $t \in(0,1], z \in \mathbb{R}$ and $v(0, z):=q_{1}(z), z \in \mathbb{R}$. Identity (5.36) implies that $v$ is another solution to (2.2) that start with initial condition $q_{1}$ at time $s$. By uniqueness, $v=u_{s, q_{1}}$. Thus $u_{s, q_{1}}(t, z)=u_{s, q_{2}}(t, z)$ for $t \in(0,1], z \in \mathbb{R}$.

\subsection{Proof of Theorem 2.2(b): Continuity of the flow.}

Proof of Theorem 2.2(b). Fix $\omega \in \Omega^{\prime}, s \geq 0, t>s, z \in \mathbb{R}$ and the initial conditions $\left(q_{n}\right)_{n \in \mathbb{Z}_{+}}$satisfying the assumptions of the theorem. It follows from the definition of $\varphi$ in Part (a) of the theorem that $\varphi\left(s, \cdot, q_{n}, \omega\right)$ is a solution to (2.2) that starts at time $s$ with the condition $q_{n}$. Since all the assumptions of Lemma 5.2 are met, we see that there exist a subsequence $\left(n_{k}\right)_{k \in \mathbb{Z}_{+}}$such that

$$
\lim _{k \rightarrow \infty} \varphi\left(s, t, q_{n_{k}}, \omega\right)(z)=\widetilde{u}(t, z, \omega)
$$

for some $\widetilde{u}$ and $\widetilde{u}(\cdot, \cdot, \omega)$ solves (2.2) that starts at time $s$ with the initial condition $q$. On the other hand, $\varphi(s, \cdot, q, \omega)$ is also a solution to (2.2) that starts at time $s$ with the initial condition $q$. Therefore, by Theorem 2.1, these solutions coincide and $\widetilde{u}(t, z, \omega)=\varphi(s, t, q, \omega)(z)$.

Thus $\left(\varphi\left(s, t, q_{n}, \omega\right)(z)\right)_{n \in \mathbb{Z}_{+}}$is a sequence of real numbers such that each subsequence of it has a converging sub-subsequence. Since all the limiting points coincide (and equal to $\varphi(s, t, q, \omega)(z)$ ), we see by the standard argument that

$$
\lim _{n \rightarrow \infty} \varphi\left(s, t, q_{n}, \omega\right)(z)=\varphi(s, t, q, \omega)(z) .
$$

6. Proof of Proposition 3.2 (moment bound). The proof of this proposition is long and tedious. Note that all the difficulties come from the fact that the function $t \mapsto V(t, z)$ is not a semimartingale; if this were the case, then an application of Itô's lemma would imply the required bound. In our proof, we were inspired by the ideas from [3], Section 4.

We begin by observing that the process $(V(\cdot, z))_{z \in \mathbb{R}}$ is stationary. Hence for any $z_{1}, z_{2} \in \mathbb{R}$

$$
\operatorname{Law}\left(V\left(\cdot, z_{1}\right), V\left(\cdot, z_{2}\right)\right)=\operatorname{Law}\left(V\left(\cdot, z_{1}-z_{2}\right), V(\cdot, 0)\right) .
$$

Therefore, it will be sufficient to establish inequality (3.5) in Proposition 3.2 only for $z_{1}=z, z_{2}=0, z \in \mathbb{R}$. Since we have assumed that $\left|z_{1}-z_{2}\right| \leq 1$, it is enough to prove (3.5) for $z_{1}=z, z_{2}=0,|z| \leq 1$.

To present the proof, we need to introduce a number of new objects. We fix $T>0$, the function $b$ that appears in the statement of Proposition 3.2 and consider the random function

$$
H(t, z, \alpha, \beta):=b^{\prime}(V(t, z)+\alpha)-b^{\prime}(V(t, 0)+\beta), \quad t \in[0, T], z, \alpha, \beta \in \mathbb{R} .
$$


Recall that it is assumed that $b$ is a bounded differentiable function with bounded derivative. Without loss of generality, we suppose that

$$
\|b\|_{\infty} \leq 1
$$

(otherwise we consider the function $\tilde{b}:=b /\|b\|_{\infty}$ instead of $b$ ). All constants that appear in this section do not depend on the function $b$ (satisfying condition (6.3)).

Fix $z \in[-1,1], \alpha, \beta \in \mathbb{R}, t_{1}, t_{2} \in[0, T], t_{1}<t_{2}$ and define a martingale $M^{t_{1}, t_{2}}=$ $\left(M_{t}^{t_{1}, t_{2}}\right)_{t_{1} \leq t \leq t_{2}}$, where

$$
M_{t}^{t_{1}, t_{2}}:=\mathrm{E}\left[\int_{t_{1}}^{t_{2}} H(r, z, \alpha, \beta) d r \mid \mathcal{F}_{t}\right], \quad t_{1} \leq t \leq t_{2}
$$

Recall that $\left(\mathcal{F}_{t}\right)_{t \geq 0}$ is the filtration associated with $\dot{W}$. By definition, for any $z \in$ $[-1,1], 0 \leq s \leq t$ the random variable $V(s, t, z, \omega)$ is $\mathcal{F}_{t}$-measurable.

Using the new notation and taking into account formula (6.1), we can rewrite the left-hand side of inequality (3.5) in Proposition 3.2 as $\mathrm{E}\left|M_{t_{2}}^{t_{1}, t_{2}}\right|^{p}$. We clearly have

$$
\mathrm{E}\left|M_{t_{2}}^{t_{1}, t_{2}}\right|^{p} \leq C \mathrm{E}\left|M_{t_{1}}^{t_{1}, t_{2}}\right|^{p}+C \mathrm{E}\left|M_{t_{2}}^{t_{1}, t_{2}}-M_{t_{1}}^{t_{1}, t_{2}}\right|^{p} .
$$

The first term in the right-hand side of (6.5) is easy to bound. Namely, we first estimate $\mathrm{E}\left[H(r, z, \alpha, \beta) \mid \mathcal{F}_{t_{1}}\right]$ for $r \in\left[t_{1}, t_{2}\right]$ (this is done in Lemma 6.2) and then apply the integral Minkowski inequality.

To estimate the second term in the right-hand side of (6.5), we first calculate the quadratic variation of the martingale $M^{t_{1}, t_{2}}$ (i.e., $\left[M^{t_{1}, t_{2}}\right]_{t}$ ) and then apply the Burkholder-Davis-Gundy inequality.

Let us briefly explain how we estimate $\left[M^{t_{1}, t_{2}}\right]_{t}$. To simplify the notation, if there is no ambiguity, further we drop the superindex $\left(t_{1}, t_{2}\right)$ and write $M_{t}$ instead of $M_{t}^{t_{1}, t_{2}}$. Recall that for continuous martingales quadratic variation [ $\left.\cdot\right]_{t}$ equals predictable quadratic variation $\langle\cdot\rangle_{t}$. To calculate $\langle M\rangle_{t}$, we use the following identity valid for $t_{1} \leq r \leq s \leq t_{2}$ :

$$
\begin{aligned}
M_{s}-M_{r}= & \int_{t_{1}}^{t_{2}} \mathrm{E}\left(\left[H(t, z, \alpha, \beta) \mid \mathcal{F}_{s}\right]-\mathrm{E}\left[H(t, z, \alpha, \beta) \mid \mathcal{F}_{r}\right]\right) d t \\
= & \int_{s}^{t_{2}} \mathrm{E}\left(\left[H(t, z, \alpha, \beta) \mid \mathcal{F}_{s}\right]-\mathrm{E}\left[H(t, z, \alpha, \beta) \mid \mathcal{F}_{r}\right]\right) d t \\
& +\int_{r}^{s} H(t, z, \alpha, \beta) d t-\int_{r}^{s} \mathrm{E}\left[H(t, z, \alpha, \beta) \mid \mathcal{F}_{r}\right] d t \\
= & : I_{1}\left(r, s, t_{2}\right)+I_{2}(r, s)-I_{3}(r, s) .
\end{aligned}
$$

Note that the terms $I_{1}, I_{2}, I_{3}$ depend also on $\alpha, \beta, z$. To simplify the notation, we omit this dependence.

Thus we can bound the term $\mathrm{E}\left(\left(M_{S}-M_{r}\right)^{2} \mid \mathcal{F}_{r}\right)$, which is the main ingredient of $\langle M\rangle_{t}$, as follows:

$$
\mathrm{E}\left(\left(M_{s}-M_{r}\right)^{2} \mid \mathcal{F}_{r}\right) \leq C \mathrm{E}\left(I_{1}\left(r, s, t_{2}\right)^{2} \mid \mathcal{F}_{r}\right)+C \mathrm{E}\left(I_{2}(r, s)^{2} \mid \mathcal{F}_{r}\right)+C I_{3}(r, s)^{2},
$$


where we have also used $\mathcal{F}_{r}$-measurability of $I_{3}(r, s)$. The corresponding bounds for the terms in right-hand side of the above inequality are obtained in Lemmas 6.2-6.4. We combine all these bounds together in Lemma 6.5.

To prove that the martingale $M$ is continuous, we split it into two parts $M:=$ $L+N$, where

$$
\begin{array}{ll}
L_{t}:=I_{2}\left(t_{1}, t\right)=\int_{t_{1}}^{t} H(r, z, \alpha, \beta) d r, & t_{1} \leq t \leq t_{2} ; \\
N_{t}:=\mathrm{E}\left[\int_{t}^{t_{2}} H(r, z, \alpha, \beta) d r \mid \mathcal{F}_{t}\right], & t_{1} \leq r \leq t_{2} .
\end{array}
$$

Since $H$ is bounded, the process $L$ is obviously continuous. To prove that $N$ is also continuous, we employ the Kolmogorov continuity theorem. We use the identity $N_{s}-N_{r}=I_{1}\left(r, s, t_{2}\right)-I_{3}(r, s)$ valid for $t_{1} \leq r \leq s \leq t_{2}$. This is done in Lemma 6.6.

Finally, we calculate quadratic variation $[M]_{t}$, which, by the continuity of $M$, is equal to $\langle M\rangle_{t}$. This is done also in Lemma 6.6. At the end of the section, we combine the obtained estimates and prove Proposition 3.2.

We begin with the following simple estimate.

LEMMA 6.1. Let b be a bounded differentiable function with bounded derivative and $\|b\|_{\infty} \leq 1$. Then for any $\delta_{1}, \delta_{2}, \delta_{3} \in[0,1]$, there exists a constant $C=$ $C\left(\delta_{1}, \delta_{2}, \delta_{3}\right)$ such that for any $a_{0}, a_{1}, a_{2}, a_{3} \in \mathbb{R}$ and any Gaussian random variable $X$ with zero mean and variance $\operatorname{Var} X=\sigma^{2}$ we have the following bounds:

$$
\begin{aligned}
& \left|\mathrm{E}\left(b^{\prime}\left(X+a_{0}+a_{1}\right)-b^{\prime}\left(X+a_{0}\right)\right)\right| \leq C \sigma^{-1-\delta_{1}}\left|a_{1}\right|^{\delta_{1}} ; \\
& \quad \leq \mathrm{E}\left(b^{\prime}\left(X+a_{0}+a_{1}+a_{2}\right)-b^{\prime}\left(X+a_{0}+a_{1}\right)-b^{\prime}\left(X+a_{0}+a_{2}\right)+b^{\prime}\left(X+a_{0}\right)\right) \mid \\
& \quad \leq C \sigma^{-1-\delta_{1}-\delta_{2}}\left|a_{1}\right|^{\delta_{1}}\left|a_{2}\right|^{\delta_{2}} ; \\
& \quad \leq C \sigma^{-1-\delta_{3}}\left|a_{1}-a_{3}\right|^{\delta_{3}}+C \sigma^{-1-\delta_{1}-\delta_{2}}\left|a_{1}\right|^{\delta_{1}}\left|a_{2}\right|^{\delta_{2}} .
\end{aligned}
$$

PROOF. First we establish bound (6.10). Fix arbitrary $\delta_{1}, \delta_{2} \in[0,1]$. We use integration by parts and rewrite the left-hand side of (6.10) in the following form:

$$
\begin{aligned}
\mid \mathrm{E} & \left(b^{\prime}\left(X+a_{0}+a_{1}+a_{2}\right)-b^{\prime}\left(X+a_{0}+a_{1}\right)-b^{\prime}\left(X+a_{0}+a_{2}\right)+b^{\prime}\left(X+a_{0}\right)\right) \mid \\
& =\left|\int_{\mathbb{R}}\left(b\left(x+a_{0}+a_{1}+a_{2}\right)-b\left(x+a_{0}+a_{2}\right)-b\left(x+a_{0}+a_{1}\right)+b\left(x+a_{0}\right)\right) p_{\sigma^{2}}^{\prime}(x) d x\right| \\
& =\left|\int_{\mathbb{R}} b\left(x+a_{0}\right)\left(p_{\sigma^{2}}^{\prime}\left(x-a_{1}-a_{2}\right)-p_{\sigma^{2}}^{\prime}\left(x-a_{2}\right)-p_{\sigma^{2}}^{\prime}\left(x-a_{1}\right)+p_{\sigma^{2}}^{\prime}(x)\right) d x\right| \\
& \leq C \sigma^{-1-\delta_{1}-\delta_{2}}\left|a_{1}\right|^{\delta_{1}}\left|a_{2}\right|^{\delta_{2}},
\end{aligned}
$$


where for the last inequality we used boundedness of the function $b$ and Lemma 4.2. Estimate (6.9) is derived by the same argument. Estimate (6.11) is a direct corollary of (6.9) and (6.10).

Now we are moving on to calculating the quadratic variation of the martingale $M$. In the next three lemmas, we will obtain a moment bound on $I_{1}$ (recall its definition in (6.6)). Recall also the definition of $\mathcal{V}$ and $H$ in (4.6) and (6.2), respectively.

Lemma 6.2. Let $\delta \in[0,1]$. There exists $C=C(T, \delta)$ such that for any $0 \leq$ $r \leq s \leq t \leq T, z, \alpha, \beta \in \mathbb{R}$ we have

$$
\begin{aligned}
&\left|\mathrm{E}\left[H(t, z, \alpha, \beta) \mid \mathcal{F}_{s}\right]-\mathrm{E}\left[H(t, z, \alpha, \beta) \mid \mathcal{F}_{r}\right]\right| \\
& \leq C(t-s)^{-1 / 2}|\mathcal{V}(r, s, t, z)-\mathcal{V}(r, s, t, 0)| \\
&+C(t-s)^{-1 / 2-\delta / 4}\left(|\mathcal{V}(0, r, t, z)-\mathcal{V}(0, r, t, 0)|^{\delta}+|\alpha-\beta|^{\delta}\right) \\
& \times(\mathrm{E}|\mathcal{V}(r, s, t, 0)|+|\mathcal{V}(r, s, t, 0)|) ;
\end{aligned}
$$

(6.13) $\left|\mathrm{E}\left[H(t, z, \alpha, \beta) \mid \mathcal{F}_{s}\right]\right|$

$$
\leq C(t-s)^{-(1+\delta) / 4}\left(|\mathcal{V}(0, s, t, z)-\mathcal{V}(0, s, t, 0)|^{\delta}+|\alpha-\beta|^{\delta}\right) .
$$

Proof. We start with the proof of inequality (6.12). Fix $0 \leq r \leq s \leq t \leq T$, $z, \alpha, \beta \in \mathbb{R}$. For $i=1,2$, introduce the following random variables:

$$
X_{i}:=\mathcal{V}\left(0, r, t, z_{i}\right) ; \quad Y_{i}:=\mathcal{V}\left(r, s, t, z_{i}\right) ; \quad Z_{i}:=\mathcal{V}\left(s, t, t, z_{i}\right),
$$

where $z_{1}:=0, z_{2}:=z$. We clearly have $V\left(t, z_{i}\right)=X_{i}+Y_{i}+Z_{i}, i=1,2$. Note also that the random vectors $\left(X_{1}, X_{2}\right),\left(Y_{1}, Y_{2}\right)$ and $\left(Z_{1}, Z_{2}\right)$ are Gaussian and independent. Additionally, $\operatorname{Law}\left(X_{1}\right)=\operatorname{Law}\left(X_{2}\right), \operatorname{Law}\left(Y_{1}\right)=\operatorname{Law}\left(Y_{2}\right)$, $\operatorname{Law}\left(Z_{1}\right)=\operatorname{Law}\left(Z_{2}\right)$. Define for $x, y \in \mathbb{R}$,

$$
J(x, y):=\mathrm{E}\left(b^{\prime}\left(x+Z_{1}\right)-b^{\prime}\left(y+Z_{1}\right)\right) .
$$

With this notation in hand, we rewrite

(6.14) $\mathrm{E}\left[H(t, z, \alpha, \beta) \mid \mathcal{F}_{s}\right]$

$$
\begin{aligned}
& =\mathrm{E}\left[b^{\prime}\left(X_{1}+Y_{1}+Z_{1}+\alpha\right)-b^{\prime}\left(X_{2}+Y_{2}+Z_{2}+\beta\right) \mid X_{1}, X_{2}, Y_{1}, Y_{2}\right] \\
& =\mathrm{E}\left[b^{\prime}\left(X_{1}+Y_{1}+Z_{1}+\alpha\right)-b^{\prime}\left(X_{2}+Y_{2}+Z_{1}+\beta\right) \mid X_{1}, X_{2}, Y_{1}, Y_{2}\right] \\
& =J\left(X_{1}+Y_{1}+\alpha, X_{2}+Y_{2}+\beta\right)
\end{aligned}
$$


and

$$
\begin{aligned}
\mathrm{E}[H & \left.(t, z, \alpha, \beta) \mid \mathcal{F}_{r}\right] \\
& =\mathrm{E}\left[b^{\prime}\left(X_{1}+Y_{1}+Z_{1}+\alpha\right)-b^{\prime}\left(X_{2}+Y_{2}+Z_{2}+\beta\right) \mid X_{1}, X_{2}\right] \\
& =\mathrm{E}\left[b^{\prime}\left(X_{1}+Y_{1}+Z_{1}+\alpha\right)-b^{\prime}\left(X_{2}+Y_{1}+Z_{1}+\beta\right) \mid X_{1}, X_{2}\right] \\
& =\left.\left[\mathrm{E} J\left(x_{1}+Y_{1}+\alpha, x_{2}+Y_{1}+\beta\right)\right]\right|_{\substack{x_{1}=X_{1} \\
x_{2}=X_{2}}} ^{\substack{y\\
}} \\
& =\int_{\mathbb{R}} J\left(X_{1}+y_{1}+\alpha, X_{2}+y_{1}+\beta\right) p_{\operatorname{Var} Y_{1}}\left(y_{1}\right) d y_{1} .
\end{aligned}
$$

Now take any $c_{1}, c_{2}, d_{1}, d_{2} \in \mathbb{R}$. We apply inequality (6.11) with the following set of parameters: $\delta_{1} \leftarrow 1, \delta_{2} \leftarrow \delta, \delta_{3} \leftarrow 1, a_{0} \leftarrow d_{2}, a_{1} \leftarrow c_{1}-d_{1}, a_{2} \leftarrow d_{1}-d_{2}$ and $a_{3} \leftarrow c_{2}-d_{2}$. We obtain

$$
\begin{aligned}
\mid J\left(c_{1}, c_{2}\right)-J\left(d_{1}, d_{2}\right) \leq & C(t-s)^{-1 / 2}\left|c_{1}-d_{1}-c_{2}+d_{2}\right| \\
& +C(t-s)^{-1 / 2-\delta / 4}\left|c_{1}-d_{1}\right|\left|d_{1}-d_{2}\right|^{\delta},
\end{aligned}
$$

where we also used the fact that

$$
\operatorname{Var} Z_{1}=\operatorname{Var} \mathcal{V}(s, t, t, 0)=C(t-s)^{1 / 2} .
$$

Combining (6.14), (6.15) and (6.16), we deduce

$$
\begin{aligned}
& \left|\mathrm{E}\left[H(t, z, \alpha, \beta) \mid \mathcal{F}_{s}\right]-\mathrm{E}\left[H(t, z, \alpha, \beta) \mid \mathcal{F}_{r}\right]\right| \\
& \quad \leq C(t-s)^{-1 / 2}\left|Y_{1}-Y_{2}\right| \\
& \quad+C(t-s)^{-1 / 2-\delta / 4}\left(\left|Y_{1}\right|+\mathrm{E}\left|Y_{1}\right|\right)\left(\left|X_{1}-X_{2}\right|^{\delta}+|\alpha-\beta|^{\delta}\right),
\end{aligned}
$$

which is (6.12).

To establish inequality (6.13), we just note that for any $c_{1}, c_{2} \in \mathbb{R}, \delta \in[0,1]$ it follows from (6.9) and (6.17) that

$$
\left|J\left(c_{1}, c_{2}\right)\right| \leq C\left(\operatorname{Var} Z_{1}\right)^{-1 / 2-\delta / 2}\left|c_{1}-c_{2}\right|^{\delta} \leq C(t-s)^{-1 / 4-\delta / 4}\left|c_{1}-c_{2}\right|^{\delta} .
$$

Combining this with (6.14), we come to (6.13).

The next statement can be called the conditional integral Minkowski inequality. This inequality is definitely not new; however, we were not able to find its proof in the literature. So we provide it here for the completeness of the argument.

LEMMA 6.3. Let $(\xi(t))_{t \geq 0}$ be a random process. Then for any $\sigma$-field $\mathcal{G} \subset \mathcal{F}$ and $0 \leq a \leq b$ we have

$$
\mathrm{E}\left[\left(\int_{a}^{b} \xi(t) d t\right)^{2} \mid \mathcal{G}\right] \leq\left(\int_{a}^{b}\left(\mathrm{E}\left[\xi(t)^{2} \mid \mathcal{G}\right]\right)^{1 / 2} d t\right)^{2}
$$


Proof. By the Fubini theorem, we have

$$
\begin{aligned}
\mathrm{E}\left[\left(\int_{a}^{b} \xi(t) d t\right)^{2} \mid \mathcal{G}\right] & =\int_{a}^{b} \int_{a}^{b} \mathrm{E}[\xi(t) \xi(s) \mid \mathcal{G}] d t d s \\
& \leq \int_{a}^{b} \int_{a}^{b}\left(\mathrm{E}\left[\xi(t)^{2} \mid \mathcal{G}\right]\right)^{1 / 2}\left(\mathrm{E}\left[\xi(s)^{2} \mid \mathcal{G}\right]\right)^{1 / 2} d t d s \\
& =\left[\int_{a}^{b}\left(\mathrm{E}\left[\xi(t)^{2} \mid \mathcal{G}\right]\right)^{1 / 2} d t\right]^{2}
\end{aligned}
$$

The next lemma provides estimates on the moments of $I_{1}$. Recall its definition in (6.6).

LEMMA 6.4. Let $p>0, \delta \in(0,1), \delta^{\prime} \in(0, \delta)$. Then there exist a random variable $K(\omega)$ and a constant $C=C\left(p, T, \delta, \delta^{\prime}\right)>0$ such that $\mathrm{E} K(\omega)^{p} \leq C$ and for any $0 \leq r \leq s \leq t \leq T, z, \alpha, \beta \in \mathbb{R},|z| \leq 1$ we have almost surely

$$
\mathrm{E}\left[I_{1}(r, s, t)^{2} \mid \mathcal{F}_{r}\right] \leq K(\omega)(s-r)(t-s)^{1 / 2-\delta / 2}\left(|z|^{\delta^{\prime}}+|\alpha-\beta|^{2 \delta}\right) .
$$

We also have for $\delta \in(0,1 / 2)$

$$
\mathrm{E}\left(I_{1}(r, s, t)\right)^{4} \leq C(s-r)^{2}(t-s)^{1-\delta}\left(|z|^{2 \delta}+|\alpha-\beta|^{4 \delta}\right) .
$$

Proof. Fix $p>0, \delta \in(0,1), \delta^{\prime} \in(0, \delta)$. We employ estimate (6.12) from Lemma 6.2 and use the corresponding estimates from Lemma 4.7 and Lemma 4.8 to obtain that there exists a random variable $K(\omega)=K\left(\omega, p, T, \delta, \delta^{\prime}\right)>0$ and a constant $C=C\left(p, T, \delta, \delta^{\prime}\right)$ such that $\mathrm{E} K(\omega)^{p} \leq C$ and

$$
\begin{array}{r}
\mathrm{E}\left[\left(\mathrm{E}\left(H\left(t^{\prime}, z, \alpha, \beta\right) \mid \mathcal{F}_{s}\right)-\mathrm{E}\left(H\left(t^{\prime}, z, \alpha, \beta\right) \mid \mathcal{F}_{r}\right)\right)^{2} \mid \mathcal{F}_{r}\right] \\
\leq K(\omega)\left(t^{\prime}-s\right)^{-3 / 2-\delta / 2}(s-r)\left(|z|^{\delta^{\prime}}+|\alpha-\beta|^{2 \delta}\right)
\end{array}
$$

for any $0 \leq r \leq s \leq t^{\prime}, z, \alpha, \beta \in \mathbb{R},|z| \leq 1$. An application of the conditional integral Minkowski inequality (Lemma 6.3) to (6.20) leads to (6.18).

Similarly, to establish bound (6.19), we also use estimate (6.12) from Lemma 6.2 and Lemma 4.8. We get

$$
\begin{gathered}
\mathrm{E}\left(\mathrm{E}\left(H\left(t^{\prime}, z, \alpha, \beta\right) \mid \mathcal{F}_{s}\right)-\mathrm{E}\left(H\left(t^{\prime}, z, \alpha, \beta\right) \mid \mathcal{F}_{r}\right)\right)^{4} \\
\leq C\left(t^{\prime}-s\right)^{-3-\delta}(s-r)^{2}\left(|z|^{2 \delta}+|\alpha-\beta|^{4 \delta}\right) .
\end{gathered}
$$

The proof is competed by an application of the integral Minkowski inequality.

We are almost ready to carry out the main goal of this subsection, that is, calculating quadratic variation of the martingale $M^{t_{1}, t_{2}}$ (recall that this martingale is defined in (6.4)). As mentioned before, a remaining technical step is to prove that this martingale is continuous. Recall that to do it we have split $M=M^{t_{1}, t_{2}}$ into two parts: $M=N+L$; see their definitions in (6.7) and (6.8). 
LEMMA 6.5. Let $p>0, \delta \in(0,1), \delta^{\prime} \in(0, \delta)$. Then there exist a random variable $K(\omega)>0$ and a constant $C=C\left(p, T, \delta, \delta^{\prime}\right)$ such that $\mathrm{E} K(\omega)^{p} \leq C$ and for any $0 \leq t_{1} \leq r \leq s \leq t_{2} \leq T, z, \alpha, \beta \in \mathbb{R},|z| \leq 1$ we have

$$
\begin{aligned}
& \text { (6.21) } \mathrm{E}\left[\left(M_{s}-M_{r}\right)^{2} \mid \mathcal{F}_{r}\right] \\
& \quad \leq K(\omega)(s-r)\left(t_{2}-r\right)^{1 / 2-\delta / 2}\left(|z|^{\delta^{\prime}}+|\alpha-\beta|^{2 \delta}\right)+8\left\|b^{\prime}\right\|_{\infty}^{2}(s-r)^{2} ; \\
& \text { (6.22) } \mathrm{E}\left(N_{s}-N_{r}\right)^{4} \leq C(s-r)^{2}\left(1+|\alpha-\beta|^{4 \delta}+\left\|b^{\prime}\right\|_{\infty}^{4}\right) .
\end{aligned}
$$

PROOF. Recall that according to our definitions (see the beginning of this section) we have

$$
\mathrm{E}\left(\left(M_{s}-M_{r}\right)^{2} \mid \mathcal{F}_{r}\right) \leq C \mathrm{E}\left(I_{1}\left(r, s, t_{2}\right)^{2} \mid \mathcal{F}_{r}\right)+C \mathrm{E}\left(I_{2}(r, s)^{2} \mid \mathcal{F}_{r}\right)+C I_{3}(r, s)^{2} .
$$

By Lemma 6.4,

$$
\mathrm{E}\left[I_{1}\left(r, s, t_{2}\right)^{2} \mid \mathcal{F}_{r}\right] \leq K(\omega)(s-r)\left(t_{2}-s\right)^{1 / 2-\delta / 2}\left(|z|^{\delta^{\prime}}+|\alpha-\beta|^{2 \delta}\right) .
$$

Note that the terms $I_{2}$ and $I_{3}$ are of order $s-r$. Therefore, they will not impact the quadratic variation. Thus we estimate them using a very rough estimate:

$$
\left|I_{2}(r, s)\right| \leq 2\left\|b^{\prime}\right\|_{\infty}(s-r) ;\left|I_{3}(r, s)\right| \leq 2\left\|b^{\prime}\right\|_{\infty}(s-r) .
$$

Hence

$$
\mathrm{E}\left[I_{2}(r, s)^{2} \mid \mathcal{F}_{r}\right]+I_{3}(r, s)^{2} \leq 8\left\|b^{\prime}\right\|_{\infty}^{2}(s-r)^{2}
$$

Thus we have

$$
\begin{aligned}
& \mathrm{E}\left[\left(M_{s}-M_{r}\right)^{2} \mid \mathcal{F}_{r}\right] \\
& \quad \leq K(\omega)(s-r)\left(t_{2}-s\right)^{1 / 2-\delta / 2}\left(|z|^{\delta^{\prime}}+|\alpha-\beta|^{2 \delta}\right)+8\left\|b^{\prime}\right\|_{\infty}^{2}(s-r)^{2}
\end{aligned}
$$

from which (6.21) follows immediately.

In a similar manner,

$$
\begin{aligned}
\mathrm{E}\left[\left(N_{s}-N_{r}\right)^{4}\right] & =\mathrm{E}\left(I_{1}\left(r, s, t_{2}\right)-I_{3}(r, s)\right)^{4} \\
& \leq C\left(\mathrm{E} I_{1}\left(r, s, t_{2}\right)^{4}+\mathrm{E} I_{3}(r, s)^{4}\right) \\
& \leq C(s-r)^{2}\left(t_{2}-s\right)^{1-\delta}\left(|z|^{2 \delta}+|\alpha-\beta|^{4 \delta}\right)+C\left\|b^{\prime}\right\|_{\infty}^{4}(s-r)^{4},
\end{aligned}
$$

where we have used (6.23) and estimate (6.19) from Lemma 6.4. This implies (6.22).

Now we have all the tools to bound the quadratic variation of $M^{t_{1}, t_{2}}$. 
LEMMA 6.6. For any $0 \leq t_{1} \leq t_{2} \leq T, z, \alpha, \beta \in \mathbb{R},|z| \leq 1$, the martingale $M^{t_{1}, t_{2}}$ defined in (6.4) is continuous. Moreover, for any $\delta \in(0,1), \delta^{\prime} \in(0, \delta)$ and any $p>0$ there exist a random variable $K(\omega)>0$ and a constant $C=$ $C\left(p, T, \delta, \delta^{\prime}\right)$ such that $\mathrm{E} K(\omega)^{p} \leq C$ and

$$
\left[M^{t_{1}, t_{2}}, M^{t_{1}, t_{2}}\right]_{t_{2}} \leq K(\omega)\left(|z|^{\delta^{\prime}}+|\alpha-\beta|^{2 \delta}\right)\left(t_{2}-t_{1}\right)^{3 / 2-\delta / 2} .
$$

PROOF. Fix $0 \leq t_{1} \leq t_{2} \leq T, z, \alpha, \beta \in \mathbb{R},|z| \leq 1$. First we prove that the martingale $M^{t_{1}, t_{2}}$ is continuous. We make use of Lemma 6.5 to obtain for $r, s \in\left[t_{1}, t_{2}\right]$,

$$
\mathrm{E}\left(N_{s}-N_{r}\right)^{4} \leq C(T, \alpha, \beta, z)\left(\left\|b^{\prime}\right\|_{\infty}^{4} \vee 1\right)(s-r)^{2} .
$$

Hence, by the Kolmogorov continuity theorem, the process $\left(N_{s}\right)_{t_{1} \leq s \leq t_{2}}$ is continuous. The process $\left(L_{s}\right)_{t_{1} \leq s \leq t_{2}}$ is also continuous since the function $H$ is bounded. Thus the martingale $M^{t_{1}, t_{2}}$ is continuous as a sum of two continuous processes.

We move on and calculate predictable quadratic variation of the martingale $M^{t_{1}, t_{2}}$. We employ Lemma 6.5 to get

$$
\begin{aligned}
\left\langle M^{t_{1}, t_{2}}, M^{t_{1}, t_{2}}\right\rangle_{t_{2}} & \\
= & \lim _{n \rightarrow \infty} \sum_{k=0}^{n-1} \mathrm{E}\left[\left(M_{t_{1}+(k+1)\left(t_{2}-t_{1}\right) / n}^{t_{1}, t_{2}}-M_{t_{1}+k\left(t_{2}-t_{1}\right) / n}^{t_{1}, t_{2}}\right)^{2} \mid \mathcal{F}_{t_{1}+k\left(t_{2}-t_{1}\right) / n}\right] \\
\leq & K(\omega)\left(|z|^{\delta^{\prime}}+|\alpha-\beta|^{2 \delta}\right)\left(t_{2}-t_{1}\right)^{3 / 2-\delta / 2} \lim _{n \rightarrow \infty} \frac{\sum_{k=0}^{n-1}(n-k)^{1 / 2-\delta / 2}}{n^{3 / 2-\delta / 2}} \\
& \quad+8\left\|b^{\prime}\right\|_{\infty}^{2}\left(t_{2}-t_{1}\right)^{2} \lim _{n \rightarrow \infty} n^{-1} \\
\leq & K(\omega)\left(|z|^{\delta^{\prime}}+|\alpha-\beta|^{2 \delta}\right)\left(t_{2}-t_{1}\right)^{3 / 2-\delta / 2} .
\end{aligned}
$$

By above, the martingale $M^{t_{1}, t_{2}}$ is continuous. Hence its quadratic variation equals its predictable quadratic variation, that is, $\left[M^{t_{1}, t_{2}}, M^{t_{1}, t_{2}}\right]_{t}=\left\langle M^{t_{1}, t_{2}}, M^{t_{1}, t_{2}}\right\rangle_{t}$. This proves (6.24).

Finally, we can prove Proposition 3.2.

Proof of Proposition 3.2. Fix $p \geq 1$. As we already pointed out at the beginning of this section, it is sufficient to show (3.5) only for $z_{1}=z, z_{2}=0$, where $|z| \leq 1$. Note also that

$$
\mathrm{E}\left(\int_{t_{1}}^{t_{2}}\left(b^{\prime}(V(u, z)+\alpha)-b^{\prime}(V(u, 0)+\beta)\right) d u\right)^{p}=\mathrm{E}\left|M_{t_{2}}^{t_{1}, t_{2}}\right|^{p} .
$$

It follows from the Burkholder-Davis-Gundy inequality and Lemma 6.6 that

$$
\begin{aligned}
\mathrm{E}\left|M_{t_{2}}^{t_{1}, t_{2}}-M_{t_{1}}^{t_{1}, t_{2}}\right|^{p} & \leq C \mathrm{E}\left[M^{t_{1}, t_{2}}, M^{t_{1}, t_{2}}\right]_{t_{2}}^{p / 2} \\
& \leq C\left(t_{2}-t_{1}\right)^{p(3 / 4-\delta / 4)}\left(|z|^{p \delta^{\prime} / 2}+|\alpha-\beta|^{p \delta}\right)
\end{aligned}
$$


where we have also used the finiteness of the $p / 2$ th moment of $K(\omega)$. By the integral Minkowski inequality,

$$
\begin{aligned}
\mathrm{E}\left|M_{t_{1}}^{t_{1}, t_{2}}\right|^{p} & =\left\|M_{t_{1}}^{t_{1}, t_{2}}\right\|_{p}^{p}=\left\|\int_{t_{1}}^{t_{2}} \mathrm{E}\left[H(t, z, \alpha, \beta) \mid \mathcal{F}_{t_{1}}\right] d t\right\|_{p}^{p} \\
& \leq\left(\int_{t_{1}}^{t_{2}}\left\|\mathrm{E}\left[H(t, z, \alpha, \beta) \mid \mathcal{F}_{t_{1}}\right]\right\|_{p} d t\right)^{p}
\end{aligned}
$$

We employ estimate (6.13) from Lemma 6.2 and Lemma 4.8 to get

$$
\left\|\mathrm{E}\left[H(t, z, \alpha, \beta) \mid \mathcal{F}_{t_{1}}\right]\right\|_{p} \leq C\left(t-t_{1}\right)^{-(1+\delta) / 4}\left(|z|^{\delta / 2}+|\alpha-\beta|^{\delta}\right) .
$$

Combining this inequality with (6.26), we obtain

$$
\mathrm{E}\left|M_{t_{1}}^{t_{1}, t_{2}}\right|^{p} \leq C\left(t_{2}-t_{1}\right)^{p(3 / 4-\delta / 4)}\left(|z|^{p \delta / 2}+|\alpha-\beta|^{p \delta}\right) .
$$

Inequality (3.5) follows now from this, (6.25) and the following simple observation:

$$
\mathrm{E}\left|M_{t_{2}}^{t_{1}, t_{2}}\right|^{p} \leq C\left(\mathrm{E}\left|M_{t_{1}}^{t_{1}, t_{2}}\right|^{p}+\mathrm{E}\left|M_{t_{2}}^{t_{1}, t_{2}}-M_{t_{1}}^{t_{1}, t_{2}}\right|^{p}\right) .
$$

The second part of Proposition 3.2 (inequality (3.6)) is established along the same lines as inequality (3.5).

\section{Proofs of Lemma 4.9 and Lemma 3.3.}

Proof of Lemma 4.9. The proof is based on Proposition 3.2 and the ideas from the proofs of [5], Lemma 3.3 and [22], Lemma 3.4. Before we begin the proof, let us just note that in the case $f_{1}=$ const, $f_{2}=$ const inequality (4.21) is almost obvious; one should just calculate the corresponding expected value and apply the Chebyshev inequality, see inequality (7.1) below. If $f_{1}$ and $f_{2}$ are piecewise constant functions, establishing (4.21) is also not very difficult. Thus, to prove (4.21) for the general case, we first establish it for a suitable piecewise-continuous approximations of $f_{1}, f_{2}$ and then pass to the limit. Let us carry out this plan.

Fix $\varepsilon>0, M>0, N \in \mathbb{N}, h>1 / 2$ and take sufficiently small $\delta$. Without loss of generality and to simplify the notation, we assume $T=1, \mu=1$; the proof for other values of $T, \mu$ is exactly the same. We will choose a specific $\delta$ later. Let $U$ be any set such that $|U| \leq \delta$. Let us verify that inequality (4.21) holds on a large enough set for all $z \in[-N, N], r \in \mathbb{N}, f_{1}, f_{2} \in \mathcal{C}^{h}(1,1, M)$. Note that by definition of the class $\mathcal{C}^{h}$, we have

$$
\sup _{\substack{z \in[-N, N] \\ t \in[0,1]}}\left|f_{i}(t, z)\right| \leq N M, \quad i=1,2 .
$$

The proof strategy relies on two observations. First we note that the random variable $V(t, z)$ has a Gaussian distribution with mean 0 and variance $\sqrt{t / \pi}$, see 
(4.18). Hence for any $x \in \mathbb{R}, z \in \mathbb{R}, 0 \leq t_{1}<t_{2} \leq 2$ we have

$$
\begin{aligned}
E \int_{t_{1}}^{t_{2}} \mathbb{1}_{U}(V(t, z)+x) d t & =\int_{t_{1}}^{t_{2}} \int_{\mathbb{R}} \mathbb{1}_{U}(y+x) p_{\sqrt{t / \pi}}(y) d y d t \\
& \leq C_{1}\left(\int_{\mathbb{R}} \mathbb{1}_{U}(y) d y\right)^{1 / 2} \int_{t_{1}}^{t_{2}} t^{-1 / 8} d t \\
& \leq C_{1} \sqrt{\delta}\left|t_{2}-t_{1}\right|^{7 / 8} .
\end{aligned}
$$

We fix a large integer $m$ (the precise value of $m$ will be chosen later) and split the interval $[0,2]$ into $2^{m+1}$ smaller subintervals. For $k \in\left[0,2^{m+1}-1\right]$, consider the event

$$
A_{m}^{k}(\varepsilon, x, z):=\left\{\int_{k 2^{-m}}^{(k+1) 2^{-m}} \mathbb{1}_{U}(V(t, z)+x) d t<\varepsilon 2^{-m}\right\} .
$$

By the Chebyshev inequality, (7.1) implies $\mathrm{P}\left(A_{k}(x, \varepsilon, z)\right) \geq 1-C_{1} \sqrt{\delta} \varepsilon^{-1} 2^{m / 8}$. Thus, for the event

$$
A_{m}(\varepsilon):=\bigcap_{k=0}^{2^{m+1}-1} \bigcap_{i=-M N 2^{4 m+1}}^{M N 2^{4 m+1}} \bigcap_{j=-N 2^{8 m}}^{N 2^{8 m}} A_{m}^{k}\left(\varepsilon, i 2^{-4 m}, j 2^{-8 m}\right)
$$

we have

$$
\mathrm{P}\left(A_{m}(\varepsilon)\right) \geq 1-C_{1} M N^{2} 2^{14 m} \sqrt{\delta} \varepsilon^{-1} .
$$

Second, we fix $\rho \in(1 / 2,1)$ and $\theta \in(0,1)$ such that

$$
\rho(h-1 / 4)-1 / 4-\theta>\theta .
$$

Since we have assumed that $h>1 / 2$, we see that such $\rho, \theta$ exist. We derive from Proposition 3.2 and the Kolmogorov continuity theorem that for $x, y \in$ $[-2 M N, 2 M N], z_{1}, z_{2} \in[-N, N], 0 \leq t_{1}<t_{2} \leq 2$ we have

$$
\begin{aligned}
& \left|\int_{t_{1}}^{t_{2}}\left(\mathbb{1}_{U}\left(V\left(t, z_{1}\right)+x\right)-\mathbb{1}_{U}\left(V\left(t, z_{2}\right)+y\right)\right) d t\right| \\
& \quad \leq K(\omega)\left(t_{2}-t_{1}\right)^{3 / 4-\rho / 4-\theta}\left(|x-y|^{\rho}+\left|z_{1}-z_{2}\right|^{\rho / 2}\right),
\end{aligned}
$$

where $\mathrm{E} K(\omega) \leq C_{2}$. Define for $\kappa>0$ an event

$$
B(\kappa):=\{K(\omega) \leq \kappa\} .
$$

The Chebyshev inequality implies that $\mathrm{P}(B(\kappa)) \geq 1-C_{2} \kappa^{-1}$. Therefore,

$$
\mathrm{P}\left(A_{m}(\varepsilon) \cap B(\kappa)\right) \geq 1-C_{1} M N^{2} 2^{14 m} \sqrt{\delta} \varepsilon^{-1}-C_{2} \kappa^{-1} .
$$

We choose now large $\kappa$ such that $C_{2} \kappa^{-1} \leq \varepsilon / 2$. 
It follows from the above definitions and a change of variables $t^{\prime}:=t+s$ in the integral that on event $A_{m}(\varepsilon) \cap B(\kappa)$ we have

$$
\int_{k 2^{-m}}^{(k+1) 2^{-m}} \mathbb{1}_{U}(V(t+s, z)+x) d t \leq 2 \cdot 2^{-m}\left(\varepsilon+\kappa 2^{-m}\right)
$$

for all $x \in[-2 M N, 2 M N], s \in[0,1], z \in[-N, N], 0 \leq k \leq 2^{m}-1$.

Now we fix $r \in \mathbb{N}, s \in[0,1], z \in[-N, N], f_{1}, f_{2} \in \mathcal{C}^{h}(1,1, M)$. Put

$$
f(t, z):=f_{1}(t, z)+\lambda_{r}\left(f_{2}\right)(t, z) .
$$

It follows from Fatou's lemma and the fact that the set $U$ is open

$$
\begin{aligned}
\int_{0}^{1} \mathbb{1}_{U}(V(t+s, z)+f(t, z)) d t & \leq \liminf _{n \rightarrow \infty} \int_{0}^{1} \mathbb{1}_{U}\left(V(t+s, z)+\lambda_{n}(f)(t, z)\right) d t \\
& =: \liminf _{n \rightarrow \infty} I_{n}
\end{aligned}
$$

On the other hand, for any $n \geq m$ we have

$$
I_{n} \leq I_{m}+\sum_{l=m}^{n-1}\left|I_{l+1}-I_{l}\right|
$$

The function $\lambda_{m}(f)$ is constant on time intervals $\left[k 2^{-m},(k+1) 2^{-m}\right)$. Moreover, since $f_{1}, f_{2} \in \mathcal{C}^{h}(1,1, M)$, we see that $\left|\lambda_{m}(f)(t, z)\right| \leq 2 N M$ for $t \in[0,1], z \in$ $[-N, N]$. Therefore, (7.4) yields that on $A_{m}(\varepsilon) \cap B(\kappa)$,

$$
\int_{0}^{1} \mathbb{1}_{U}\left(V(t+s, z)+\lambda_{m}(f)(t, z)\right) d t \leq 2 \varepsilon+\kappa 2^{-m+1} .
$$

In a similar way, we estimate the second term in the right-hand side of (7.6). It follows from the definition of the approximation operator $\lambda$ that if $l \geq r$, then $\lambda_{r}\left(f_{2}\right)\left((i+1) 2^{-l}, z\right)=\lambda_{r}\left(f_{2}\right)\left((i+1 / 2) 2^{-l}, z\right)$ for any $i=0,1, \ldots, 2^{l}-1$; further, if $l<r$, then $\lambda_{r}\left(f_{2}\right)\left((i+1) 2^{-l}, z\right)=f_{2}\left((i+1) 2^{-l}, z\right)$ and we have $\lambda_{r}\left(f_{2}\right)\left((i+1 / 2) 2^{-l}, z\right)=f_{2}\left((i+1 / 2) 2^{-l}, z\right)$. This observation, the definition of the set $B(\kappa)$ and a change of variables $t^{\prime}:=t+s$ in the integral imply that for $l \in \mathbb{Z}_{+}$on $A_{m}(\varepsilon) \cap B(\kappa)$,

$$
\begin{aligned}
\left|I_{l+1}-I_{l}\right| & \leq \kappa 2^{-l(3 / 4-\rho / 4-\theta)} \sum_{i=0}^{2^{l}-1}\left|f\left((i+1) 2^{-l}, z\right)-f\left((i+1 / 2) 2^{-l}, z\right)\right|^{\rho} \\
& \leq \kappa M N 2^{-l(3 / 4-5 \rho / 4+h \rho-\theta)} \sum_{i=0}^{2^{l}-1}(i+1 / 2)^{-\rho} \\
& \leq 2(1-\rho)^{-1} \kappa M N 2^{-l(\rho(h-1 / 4)-1 / 4-\theta)} \\
& \leq 2(1-\rho)^{-1} \kappa M N 2^{-l \theta}
\end{aligned}
$$


where in the last inequality we took into the account that $\rho$ and $\theta$ were chosen according to (7.2). Combining this with the previous estimate and (7.6), we finally get on $A_{m}(\varepsilon) \cap B(\kappa)$ for any $n \geq m$,

$$
\begin{aligned}
& \int_{0}^{1} \mathbb{1}_{U}\left(V(t+s, z)+\lambda_{n}(f)(t, z)\right) d t \\
& \quad \leq 2 \varepsilon+\kappa 2^{-m+1}+2(1-\rho)^{-1} \kappa M N 2^{-m \theta}\left(1-2^{-\theta}\right)^{-1} .
\end{aligned}
$$

Recall that we have already chosen $\kappa, \rho, \theta$. Now we choose large $m$ such that the right-hand side of the above inequality is less than $3 \varepsilon$. Finally, we choose small $\delta$ such than the right-hand side of (7.3) is bigger than $1-\varepsilon$.

Thus we got that on the set $D:=A_{m}(\varepsilon) \cap B(\kappa)$ we have for any $n \geq m$

$$
\int_{0}^{1} \mathbb{1}_{U}\left(V(t+s, z)+\lambda_{n}(f)(t, z)\right) d t \leq 3 \varepsilon
$$

and $P(D) \geq 1-\varepsilon$. This inequality together with (7.5) yields the statement of the lemma.

PROOF OF LEMMA 3.3. The lemma is proved by a straightforward application of Lemma 4.9.

Acknowledgments. The authors are very grateful to Siva Athreya, Haya Kaspi, Andrew Lawrie, Eyal Neuman and Alexander Shaposhnikov for useful discussions. The authors would also like to thank the referees for their valuable comments and suggestions which helped to improve the quality of the paper.

OB is very grateful to MSRI for their support and hospitality.

\section{SUPPLEMENTARY MATERIAL}

Supplement to "Regularization by noise and flows of solutions for a stochastic heat equation" (DOI: 10.1214/18-AOP1259SUPP; .pdf). The supplementary material provides proofs of auxiliary results related to the properties of the heat kernel.

\section{REFERENCES}

[1] Bally, V., Gyöngy, I. and PARdouX, É. (1994). White noise driven parabolic SPDEs with measurable drift. J. Funct. Anal. 120 484-510. MR1266318

[2] Butkovsky, O. and Mytnik, L. (2019). Supplement to "Regularization by noise and flows of solutions for a stochastic heat equation.” DOI:10.1214/18-AOP1259SUPP.

[3] Catellier, R. and Gubinelli, M. (2016). Averaging along irregular curves and regularisation of ODEs. Stochastic Process. Appl. 126 2323-2366. MR3505229

[4] CERrai, S. (2003). Stochastic reaction-diffusion systems with multiplicative noise and nonLipschitz reaction term. Probab. Theory Related Fields 125 271-304. MR1961346

[5] Davie, A. M. (2007). Uniqueness of solutions of stochastic differential equations. Int. Math. Res. Not. IMRN 2007 Art. ID rnm124, 26. MR2377011 
[6] FedrizZI, E. and Flandoli, F. (2013). Hölder flow and differentiability for SDEs with nonregular drift. Stoch. Anal. Appl. 31 708-736. MR3175794

[7] Flandoli, F. (1995). Regularity Theory and Stochastic Flows for Parabolic SPDEs. Stochastics Monographs 9. Gordon and Breach Science Publishers, Yverdon. MR1347450

[8] Flandoli, F. (2011). Random Perturbation of PDEs and Fluid Dynamic Models. Lecture Notes in Math. 2015. Springer, Heidelberg. MR2796837

[9] Flandoli, F., Gubinelli, M. and Priola, E. (2010). Well-posedness of the transport equation by stochastic perturbation. Invent. Math. 180 1-53. MR2593276

[10] Folland, G. B. (1999). Real Analysis: Modern Techniques and Their Applications, 2nd ed. Wiley, New York. MR1681462

[11] Goldys, B. and ZhANG, X. (2011). Stochastic flows for nonlinear SPDEs driven by linear multiplicative space-time white noises. In Stochastic Analysis with Financial Applications. Progress in Probability 65 83-97. Birkhäuser/Springer Basel AG, Basel. MR3050785

[12] GYÖNGY, I. and PARDOUX, É. (1993). On quasi-linear stochastic partial differential equations. Probab. Theory Related Fields 94 413-425. MR1201552

[13] GyÖNGY, I. and PARDouX, É. (1993). On the regularization effect of space-time white noise on quasi-linear parabolic partial differential equations. Probab. Theory Related Fields $\mathbf{9 7}$ 211-229. MR1240724

[14] Hairer, M. and PardouX, É. (2015). A Wong-Zakai theorem for stochastic PDEs. J. Math. Soc. Japan 67 1551-1604. MR3417505

[15] HU, Y. and LE, K. (2013). A multiparameter Garsia-Rodemich-Rumsey inequality and some applications. Stochastic Process. Appl. 123 3359-3377. MR3071383

[16] Khoshnevisan, D. (2009). A primer on stochastic partial differential equations. In A Minicourse on Stochastic Partial Differential Equations. Lecture Notes in Math. 1962 1-38. Springer, Berlin. MR2508772

[17] KRYLOV, N. V. and RÖCKNER, M. (2005). Strong solutions of stochastic equations with singular time dependent drift. Probab. Theory Related Fields 131 154-196. MR2117951

[18] Kunita, H. (1997). Stochastic Flows and Stochastic Differential Equations. Cambridge Studies in Advanced Mathematics 24. Cambridge Univ. Press, Cambridge. MR1472487

[19] Mohammed, S.-E. A., Nilssen, T. K. and Proske, F. N. (2015). Sobolev differentiable stochastic flows for SDEs with singular coefficients: Applications to the transport equation. Ann. Probab. 43 1535-1576. MR3342670

[20] Priola, E. (2018). Davie's type uniqueness for a class of SDEs with jumps. Ann. Inst. H. Poincaré Probab. Statist. 54 694-725. MR3795063

[21] RezaKhanlou, F. (2014). Regular flows for diffusions with rough drifts. Preprint. Available at arXiv:1405.5856.

[22] Shaposhnikov, A. V. (2016). Some remarks on Davie's uniqueness theorem. Proc. Edinb. Math. Soc. (2) 59 1019-1035. MR3570126

[23] Veretennikov, A. J. (1980). Strong solutions and explicit formulas for solutions of stochastic integral equations. Mat. Sb. (N.S.) 111(153) 434-452, 480. MR0568986

[24] Walsh, J. B. (1986). An introduction to stochastic partial differential equations. In École D'été de Probabilités de Saint-Flour, XIV-1984. Lecture Notes in Math. 1180 265-439. Springer, Berlin. MR0876085

[25] Wresch, L. (2017). Path-by-path uniqueness of infinite-dimensional stochastic differential equations. Preprint. Available at arXiv:1706.07720.

[26] ZHANG, X. (2011). Stochastic homeomorphism flows of SDEs with singular drifts and Sobolev diffusion coefficients. Electron. J. Probab. 16 1096-1116. MR2820071

[27] Zvonkin, A. K. (1974). A transformation of the phase space of a diffusion process that will remove the drift. Mat. Sb. (N.S.) 93(135) 129-149, 152. MR0336813 
FACUlTy of INDUSTRIAL ENGINEERING AND MANAGEMENT TECHNION-ISRAEL INSTITUTE OF TECHNOLOGY HAIFA 3200003

ISRAEL

E-MAIL: oleg.butkovskiy@gmail.com leonid@technion.ac.il 